\title{
Endoplasmic reticulum stress sensor IRE1a propels neutrophil hyperactivity in lupus
}

\author{
Gautam Sule, ${ }^{1}$ Basel H. Abuaita, ${ }^{2}$ Paul A. Steffes, ${ }^{1}$ Andrew T. Fernandes, ${ }^{1}$ Shanea K. Estes, ${ }^{1}$ Craig Dobry, ${ }^{3}$ Deepika Pandian, \\ Johann E. Gudjonsson, ${ }^{3}$ J. Michelle Kahlenberg, ${ }^{1}$ Mary X. O'Riordan, ${ }^{2}$ and Jason S. Knight ${ }^{1}$
}

'Division of Rheumatology, Department of Internal Medicine, ${ }^{2}$ Department of Microbiology and Immunology, and ${ }^{3}$ Department of Dermatology, University of Michigan, Ann Arbor, Michigan, USA.

\begin{abstract}
Neutrophils amplify inflammation in lupus through the release of neutrophil extracellular traps (NETs). The endoplasmic reticulum stress sensor inositol-requiring enzyme $1 \alpha$ (IRE1 $\alpha$ ) has been implicated as a perpetuator of inflammation in various chronic diseases; however, IRE1 $\alpha$ has been little studied in relation to neutrophil function or lupus pathogenesis. Here, we found that neutrophils activated by lupus-derived immune complexes demonstrated markedly increased IRE1 $\alpha$ ribonuclease activity. Importantly, in neutrophils isolated from patients with lupus, we also detected heightened IRE1 $\alpha$ activity that was correlated with global disease activity. Immune complex-stimulated neutrophils produced both mitochondrial ROS (mitoROS) and the activated form of caspase-2 in an IRE1 $\alpha$-dependent fashion, whereas inhibition of IRE1 $\alpha$ mitigated immune complex-mediated NETosis (in both human neutrophils and a mouse model of lupus). Administration of an IRE1 $\alpha$ inhibitor to lupus-prone MRL/Ipr mice over 8 weeks reduced mitoROS levels in peripheral blood neutrophils, while also restraining plasma cell expansion and autoantibody formation. In summary, these data identify a role for IRE1 $\alpha$ in the hyperactivity of lupus neutrophils and show that this pathway is upstream of mitochondrial dysfunction, mitoROS formation, and NETosis. We believe that inhibition of the IRE1 $\alpha$ pathway is a novel strategy for neutralizing NETosis in lupus, and potentially other inflammatory conditions.
\end{abstract}

\section{Introduction}

Systemic lupus erythematosus (SLE), which is commonly referred to as lupus, is a prototypical systemic autoimmune disease. The immunopathology of lupus is complex, with derangements present in both lymphoid- and myeloid-lineage cells (1). Neutrophils and neutrophil extracellular traps (NETs) - tangles of chromatin and microbicidal proteins expelled from neutrophils in response to both infectious and sterile stimuli $(2,3)$ - play several roles in the pathophysiology of lupus (4-6). As examples, NETs promote type 1 IFN production $(7,8)$, autoantibody formation $(9,10)$, endothelial damage (11), and thrombosis $(12,13)$. At the same time, autoantibodies including anti-ribonucleoprotein (anti-RNP) (8), anti-LL-37 (14), anti-phospholipid (13), and anti-dsDNA (15) promote further NETosis, thereby setting up a vicious cycle.

While much remains to be learned about the cellular biology of NETosis, ROS formation appears to be required in most contexts (3). Importantly, neutrophil ROS are generated not only by the NADPH oxidase complex at cellular and phagosome membranes, but also by mitochondrial metabolism as a byproduct of aerobic respiration. Such mitochondrial ROS (mitoROS) likely play a particularly important role when NETs are released in response to physiologic stimuli

Conflict of interest: JEG received research funding from AbbVie, SunPharma, Celgene, and Genentech and serves on advisory boards for Novartis, AbbVie, and MiRagen JMK received research funding from Celgene and serves on advisory boards for AstraZeneca, Boehringer Ingelheim, Bristol Myers Squibb, and Eli Lilly. Copyright: ( 2021, American Society for Clinical Investigation. Submitted: March 5, 2020; Accepted: February 5, 2021; Published: April 1, 2021 Reference information: J Clin Invest. 2021;131(7):e137866. https://doi.org/10.1172/JCl137866. (or their mimickers), such as cholesterol, Staphylococcus aureus, and lupus autoantibodies $(7,16,17)$. In lupus, the formation of mitoROS by lupus neutrophils drives mitochondrial DNA (mitoDNA) oxidation and thereby potentiates the release of IFN-promoting NETs (7, $18,19)$. However, a barrier to leveraging these findings therapeutically is that the upstream regulators of mitochondrial stress and mitoROS in lupus neutrophils remain largely undefined.

The ER is an endomembrane compartment highly sensitive to inflammatory and oxidative perturbation. Indeed, beyond the ER's responsibility for synthesis and processing of membrane and secreted proteins, it also regulates calcium ion flux, the formation of autophagocytic vesicles, and the relay of oxidative and inflammatory signals (20). Key sensors of ER stress include inositol-requiring enzyme $1 \alpha$ (IRE1 $\alpha$ ), PKR-like endoplasmic reticulum kinase (PERK), and cyclic AMP-dependent transcription factor (ATF6) (21). IRE1 $\alpha$ signaling is the most phylogenetically conserved branch of the ER stress response and the best studied in terms of its intersection with inflammatory pathways. For example, IRE1 $\alpha$ may be activated by TLR2 and TLR4 in macrophages, TLR7 in DCs, and TLR9 in B cells (22-24). IRE1 $\alpha$ oligomerizes upon activation, facilitating trans-autophosphorylation and unmasking a unique cytosolic endoribonuclease (RNase) activity. One of the consequences of IRE1 $\alpha$ 's RNase activity is the splicing of X-box-binding protein 1 (XBP1) mRNA, allowing translation of the active XBP1 transcription factor $(20,21)$. In parallel, and independent of its RNase activity, phosphorylated IRE1 $\alpha$ directly binds the adaptor protein TRAF2, with subsequent activation of NF- $\mathrm{B}$ and c-Jun N-terminal kinases (JNK) pathways $(20,21)$.

Little was known about IRE1 $\alpha$ in neutrophils prior to a study in 2010 that linked ER stress to neutrophil apoptosis (25). In 2015, 
A

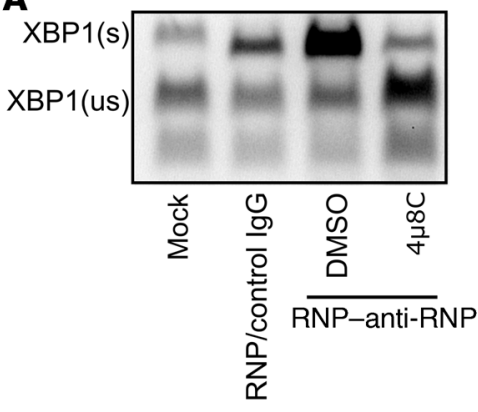

C

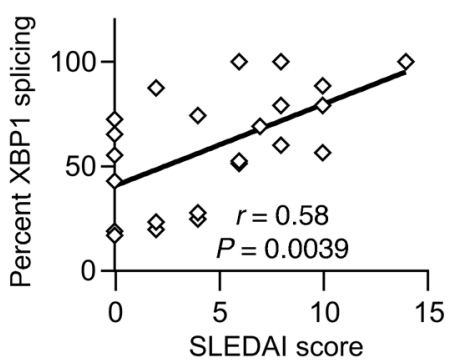

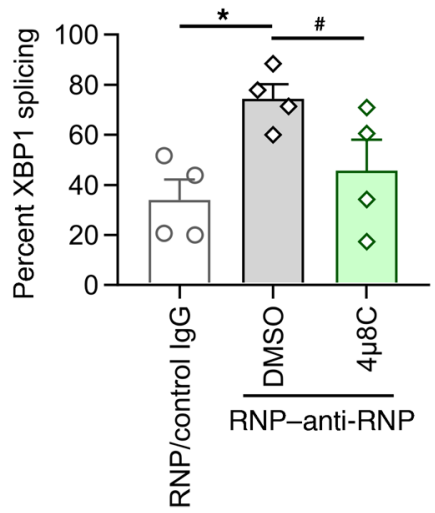

D

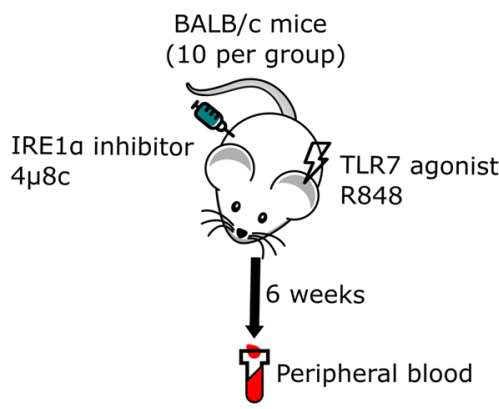

B
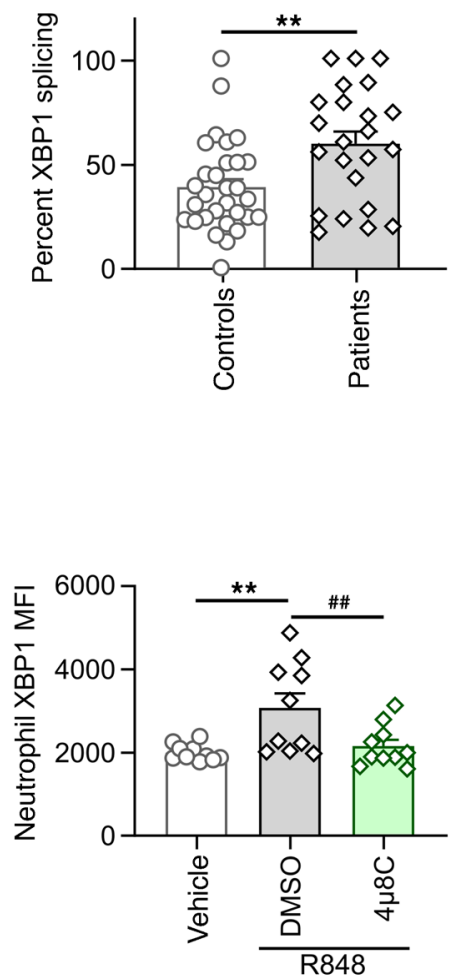

Figure 1. Activation of IRE1 $\alpha$ in lupus neutrophils. (A) Freshly isolated neutrophils with or without $4 \mu 8 \mathrm{C}$ treatment were stimulated with RNP-anti-RNP for 4 hours, and XBP1 splicing was assessed by reverse transcription qPCR, followed by Pstl digestion to cleave the unspliced product. Spliced (s) and unspliced (us) band intensities were quantified using Image J. $n=4$ independent biological replicates. ${ }^{*} P<0.05$ and ${ }^{*} P<0.05$, by 1 -way ANOVA followed by Holm-Sidak's multiple-comparison test. (B) Quantification of XBP1 splicing in neutrophils from patients with lupus. $n=23-30$ patients and healthy controls. ${ }^{* *} P<0.01$, by unpaired $t$ test. (C) Correlation between the levels of spliced XBP1 and SLEDAI scores for patients with lupus. $n=23$ patients. Correlation analysis was by Pearson's method. (D) BALB/c mice were treated with R848 and $4 \mu 8 C$ as described in Methods. BALB/c peripheral blood neutrophils were analyzed by flow cytometry for XBP1 protein indicative of spliced $X B P 1$ mRNA. $n=10$ mice per group. ${ }^{* *} P<0.01$ and ${ }^{\# \# ~} P<0.01$, by 1 -way ANOVA followed by Holm-Sidak's multiple-comparison test, compared with the DMSO control in R848 mice.

studies revealed that there is crosstalk between IRE1 $\alpha$ and NADPH oxidase-generated ROS (26) and that IRE1 $\alpha$ is required for neutrophil degranulation in a model of lung injury (27). Although research groups have focused on IRE1 $\alpha$ as a therapeutic target in inflammatory models of atherosclerosis (28), inflammatory arthritis (29), and autoimmune diabetes (30), this pathway has not been characterized in lupus neutrophils, nor has it been interrogated as a therapeutic target in lupus.

In this study, we identified IRE1 $\alpha$ as a critical mediator of immune complex-mediated NETosis in vitro. Furthermore, we found that pharmacological inhibition of IRE1 $\alpha$ reduced mitoROS formation and NET release in multiple lupus mouse models. Collectively, our data demonstrate that inhibition of IRE1 $\alpha$ may be an effective strategy for restraining mitoROS, mitoDNA oxidation, and NET formation in lupus.

\section{Results}

Increased IRE1 $\alpha$ activation in lupus neutrophils. To assess whether IRE1 $\alpha$ might be activated in the context of lupus, neutrophils isolated from healthy volunteers were stimulated with RNP-containing immune complexes (RNP-anti-RNP), and IRE1 $\alpha$ endoribonuclease activity was determined. We detected increased levels of spliced
XBP1 mRNA in neutrophils treated with RNP-anti-RNP (Figure 1A), indicating more endoribonuclease activity from activated IRE1 $\alpha$. As expected, treatment of neutrophils with the IRE1 $\alpha$ endoribonuclease inhibitor $4 \mu 8 \mathrm{C}$ reduced XBP1 splicing (Figure $1 \mathrm{~A}$ ). We assessed IRE1 $\alpha$ activation in patients with lupus via characterization of neutrophils isolated from 23 patients with lupus (92\% female, average age 49 years) and 30 healthy volunteers ( $51 \%$ female, average age 47 years) to determine the levels of spliced XBP1. We detected enhanced XBP1 splicing (indicating increased IRE1 $\alpha$ endoribonuclease activity) in neutrophils isolated from patients with lupus as compared with levels in healthy volunteers (Figure 1B). Furthermore, we found a significant positive correlation between lupus the SLEDAI (SLE disease activity index) score (activity of lupus disease) and the extent of XBP1 splicing (Figure 1C). Although we also detected increased levels of calprotectin in lupus plasma, these levels did not demonstrate a statistically significant correlation with neutrophil XBP1 splicing (Supplemental Figure 1; supplemental material available online with this article; https://doi.org/10.1172/ JCI137866DS1). To further extend these studies to the in vivo setting, we tested the endoribonuclease activity of IRE1 $\alpha$ in an inducible lupus mouse model (Figure 1D) involving epicutaneous application of the TLR7/8 agonist R848 to wild-type BALB/c mice (R848 
A

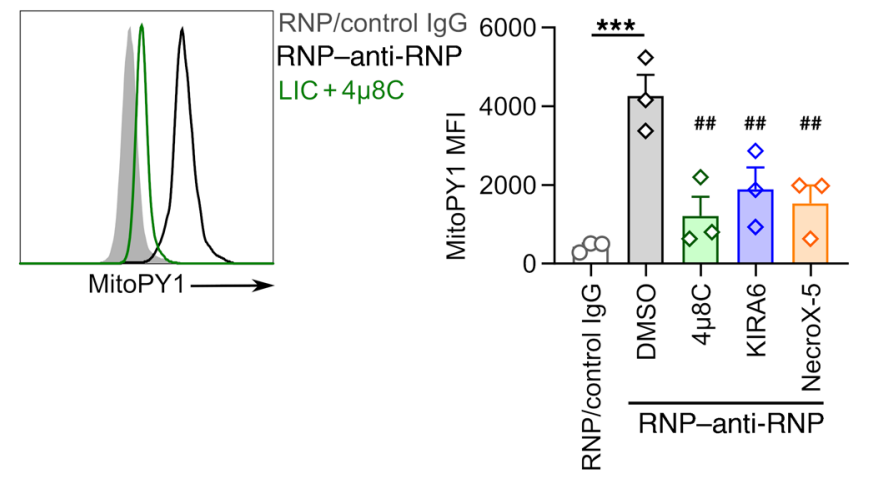

B

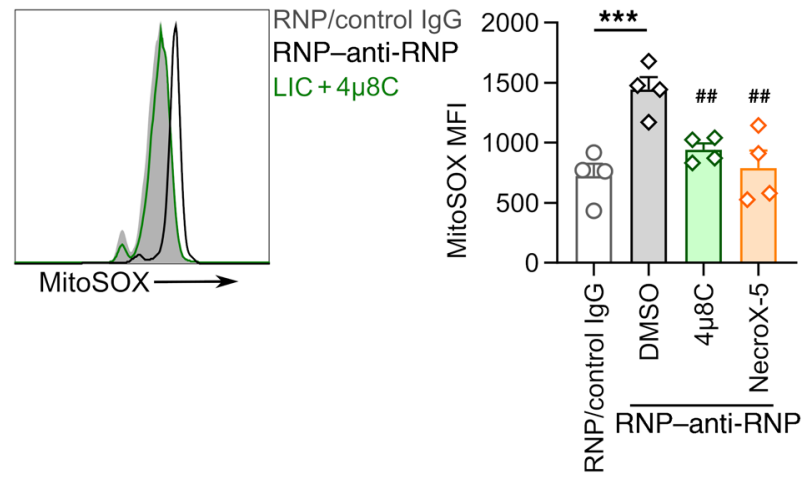

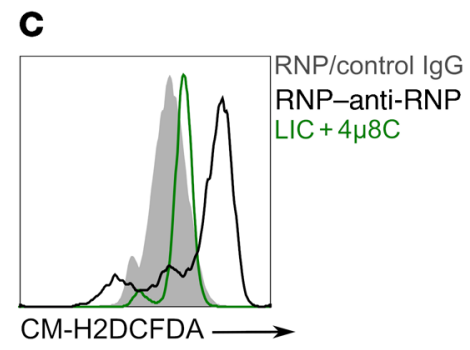

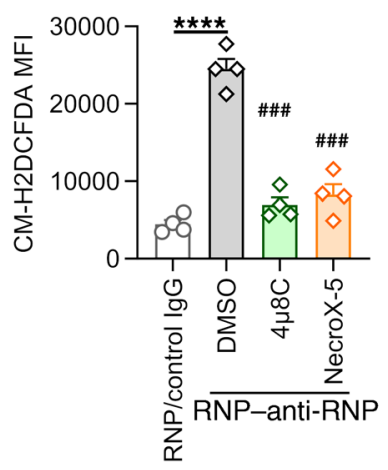

D
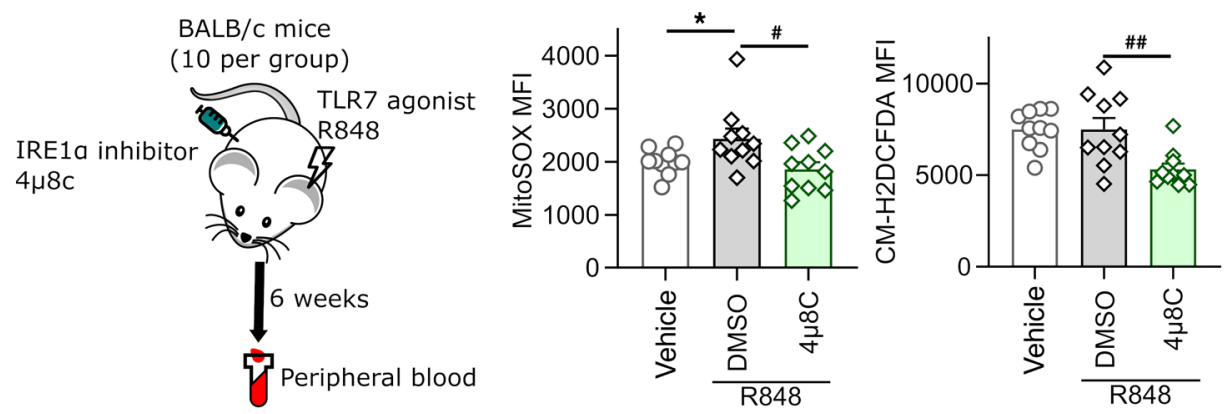

Figure 2. mitoROS generation is potentiated by IRE1 $\alpha$. Neutrophils from healthy volunteers were stimulated as indicated in the presence of IRE1 $\alpha$ inhibitors (4 $\mu 8$ C, KIRA6) or the mitoROS scavenger NecroX-5. (A) MitoPY1 and (B) mitoROS (MitoSOX) were quantified by flow cytometry. Representative histograms and quantifications are shown. $n=3$ independent biological replicates for MitoPY1; $n=4$ independent biological replicates for MitoSOX. ${ }^{* * *} P$ $<0.001$ and ${ }^{\# \# P}<0.01$, compared with the RNP-anti-RNP (DMSO) group, by 1-way ANOVA followed by Holm-Sidak's multiple-comparison test. (C) Total

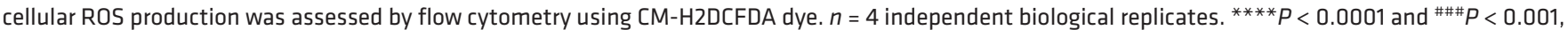
compared with the RNP-anti-RNP (DMSO) group, by 1-way ANOVA followed by Holm-Sidak's multiple-comparison test. (D) BALB/c mice were treated with R848 and the IRE1 $\alpha$ inhibitor 4 $\mu 8 \mathrm{C}$ as described in Methods. mitoROS (MitoSOX) and total cellular ROS (CM-H2DCFDA) were measured in peripheral blood neutrophils by flow cytometry. $n=10$ mice per group. ${ }^{*} P<0.05$, ${ }^{\#} P<0.05$, and ${ }^{\# \#} P<0.01$, by 1-way ANOVA followed by Holm-Sidak's multiple-comparison test, compared with the DMSO control in R848 mice.

mice) (31). As compared with control mice, peripheral blood neutrophils isolated from R848 mice demonstrated elevated levels of XBP1 protein, thereby indicating increased IRE1 $\alpha$ activity (Figure 1D). Importantly, the levels of XBP1 returned to control levels upon preemptive administration of $4 \mu 8 \mathrm{C}(10 \mathrm{mg} / \mathrm{kg} /$ day $)$ to the $\mathrm{R} 848$ mice (Figure 1D). Taken together, these data demonstrate increased IRE1 $\alpha$ endoribonuclease activity in lupus neutrophils.

IRE1 $\alpha$ activity promotes mitoROS generation. In lupus neutrophils, ROS generation is likely a prerequisite for the release of NETs. To assess the potential role of IRE1 1 in ROS generation, we stimulated neutrophils with RNP-anti-RNP and then measured both mitoROS and total ROS levels by flow cytometry. Compared with controls, we found that mitochondrial hydrogen peroxide $\left(\mathrm{mitoH}_{2} \mathrm{O}_{2}\right)$ levels increased upon stimulation with RNP-anti-RNP as determined with the fluorescent probe MitoPY1 (Figure 2A). Pretreatment of neutrophils with either $4 \mu 8 \mathrm{C}$ or the pan-IRE1 $\alpha$ inhibitor KIRA6 significantly reduced $\mathrm{mitoH}_{2} \mathrm{O}_{2}$ production. As a control, we treated neutrophils with the mitoROS-specific scavenger NecroX-5, which also reduced $\mathrm{mitoH}_{2} \mathrm{O}_{2}$ levels. These data were confirmed with a second mitoROS indicator dye, MitoSOX Red, with very similar results (Fig- 
A

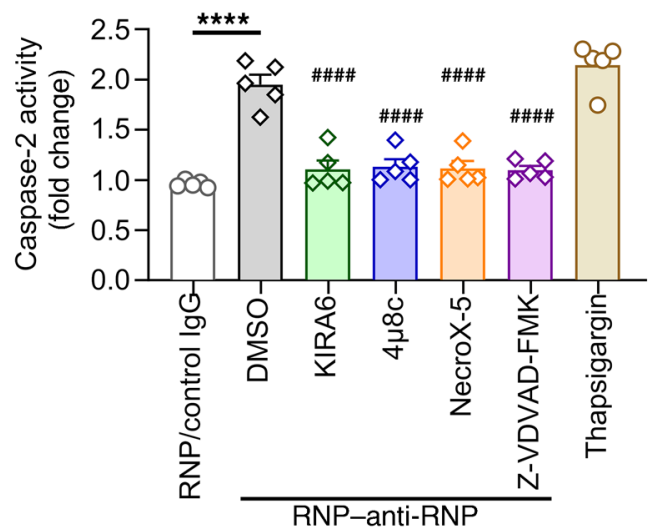

C
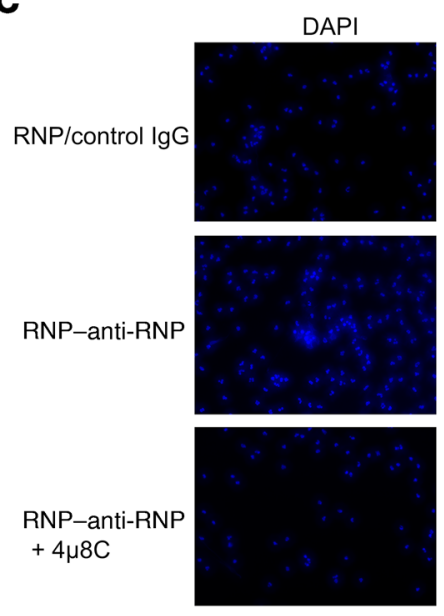

B
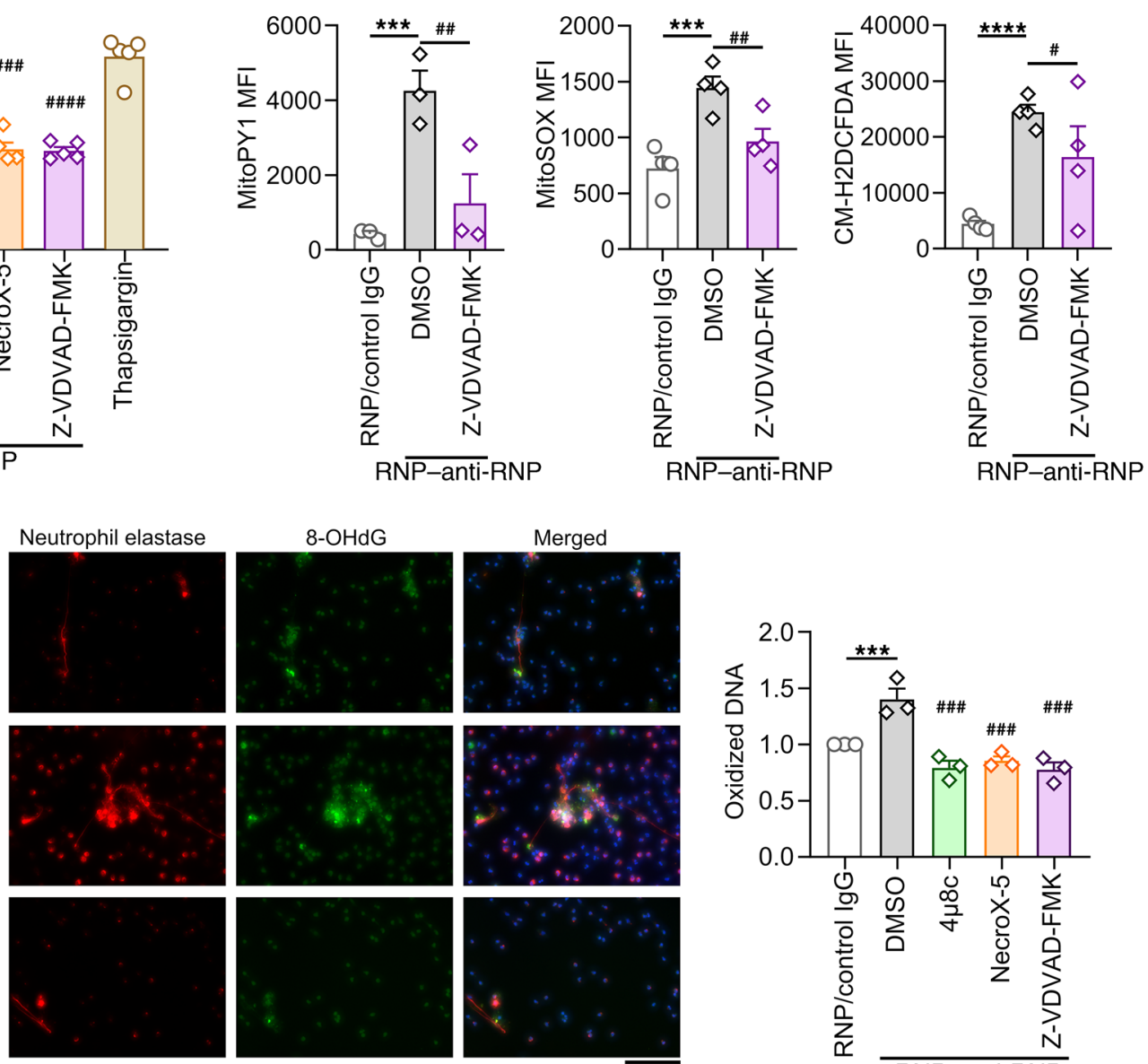

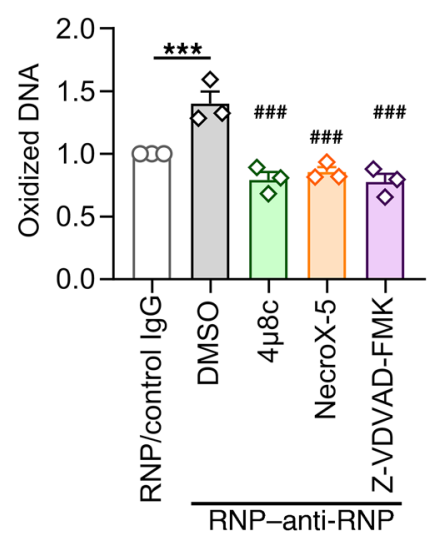

Figure 3. IRE1 $\alpha$-dependent caspase-2 activation mediates ROS generation. Control neutrophils from healthy volunteers were stimulated as indicated, including with the caspase-2 inhibitor Z-VDVAD-FMK and the mitoROS scavenger NecroX-5. (A) Caspase-2 activity was monitored by measuring the fluorescence of the probe FAM-VDVAD-FMK (indicator of the active form of caspase-2). Quantification of the probe fluorescence is presented as the fold

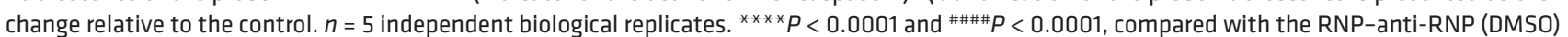
group, by 1-way ANOVA followed by Holm-Sidak's multiple-comparison test. (B) mitoROS (MitoPY1 and MitoSOX) and total cellular ROS (CM-H2DCFDA) were assayed as described in Methods. $n=3-4$ independent experiments. ${ }^{* * *} P<0.001,{ }^{* * *} P<0.0001,{ }^{*} P<0.05$, and ${ }^{\# \#} P<0.001$, by 1 -way ANOVA followed by Holm-Sidak's multiple-comparison test. (C) Neutrophils from healthy donors were stimulated as specified and stained with antibodies against 8-OHdG (green) and neutrophil elastase (red). DAPI (blue) was used to detect DNA. $n=3$ independent experiments. ${ }^{* *} P<0.001$ and ${ }^{\# \#} P<0.001$, compared with the RNP-anti-RNP (DMSO) group, by 1-way ANOVA followed by Holm-Sidak's multiple-comparison test.

ure 2B). Analogous to mitoROS levels, we found that total ROS levels increased upon RNP-anti-RNP stimulation and decreased upon treatment with $4 \mu 8 \mathrm{C}$ (Figure $2 \mathrm{C}$ ). Furthermore, in mice, inhibition of IRE1 $\alpha$ with $4 \mu 8 \mathrm{C}$ resulted in decreased levels of both mitoROS and total ROS in peripheral blood neutrophils (Figure 2D). Taken together, these data suggest that, in the context of lupus, IRE1 $\alpha$ activity contributes to ROS production by neutrophils.

IRE1 $\alpha$ activates caspase-2, which is required for efficient ROS generation. Previous work by our group revealed a role for caspase- 2 in the potentiation of mitoROS generation by activated macrophages (32). To elucidate the potential role of caspase-2 in regulating the hyperactivity of lupus neutrophils, we cultured control neutrophils with RNP-anti-RNP as described above. Stimulation with RNP-anti-RNP resulted in increased activation of caspase-2, which could be neutralized by treatment with either IRE1 $\alpha$ inhibitors $(4 \mu 8 \mathrm{C}, \mathrm{KIRA})$ ) or the specific caspase-2 inhibitor (Z-VD-
VAD-FMK) (Figure 3A and Supplemental Figure 2A). Treatment with the mitoROS scavenger NecroX-5 also dampened caspase-2 activation (Figure $3 \mathrm{~A}$ and Supplemental Figure 2A). Since caspase-2 activation has sometimes been linked to caspase-3/7mediated apoptosis $(33,34)$, we also monitored caspase-3/7 activity. In contrast to caspase-2, we did not observe significant caspase-3/7 activity upon stimulation with RNP-anti-RNP (Supplemental Figure 2B). At the same time, the caspase-2 inhibitor (Z-VDVAD-FMK) reduced neutrophil mitoROS and total ROS levels in the context of RNP-anti-RNP stimulation (Figure 3B). Since elevated ROS levels are associated with increased DNA oxidation, an 8-Oxo-2'-deoxyguanosine-specific (8-OHdG-specific) antibody was used to visualize oxidized DNA by fluorescence microscopy. We detected high levels of 8-OHdG in neutrophils cultured with RNP-anti-RNP. The levels of 8-OHdG were reduced upon treatment with either $4 \mu 8 \mathrm{C}$ or Z-VDVAD-FMK (Figure $3 \mathrm{C}$ ). In 
A

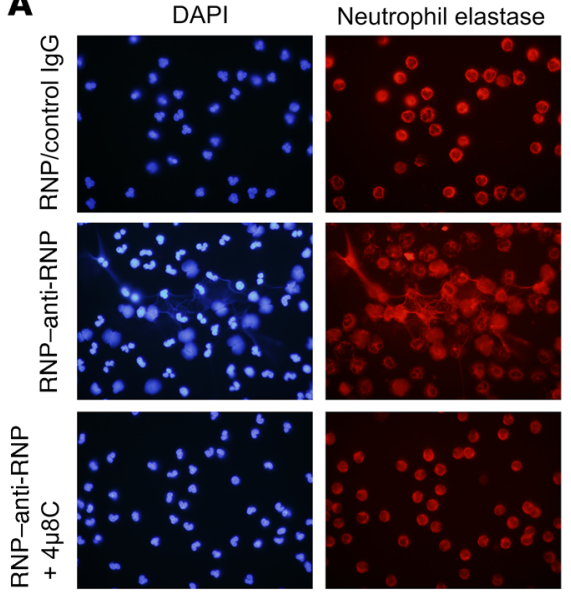

B

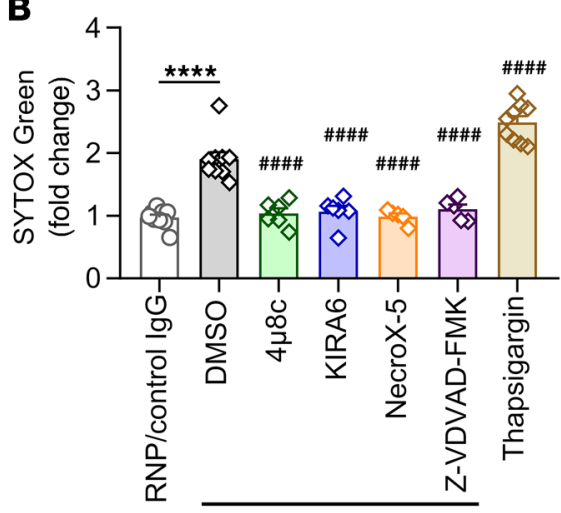

RNP-anti-RNP

D

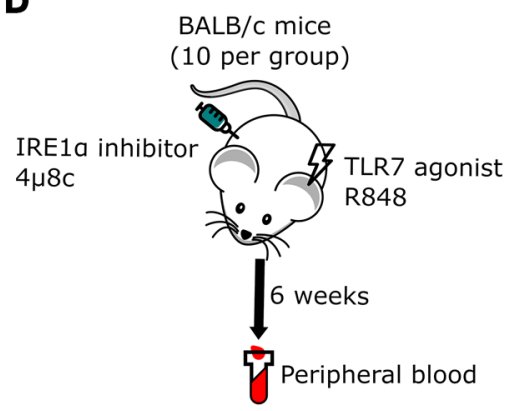

E

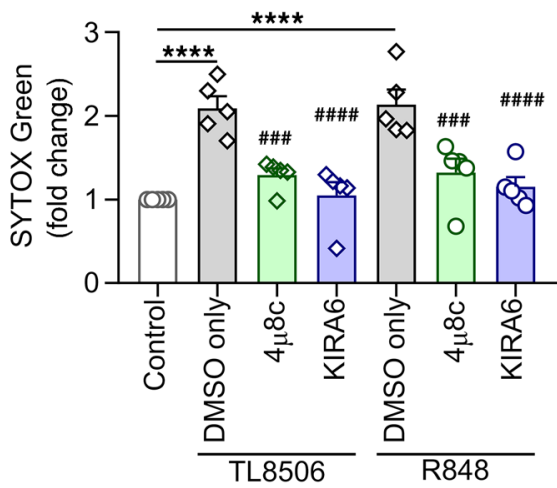

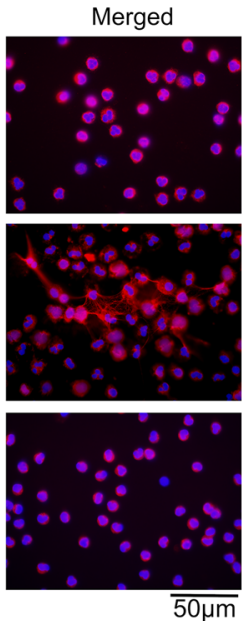

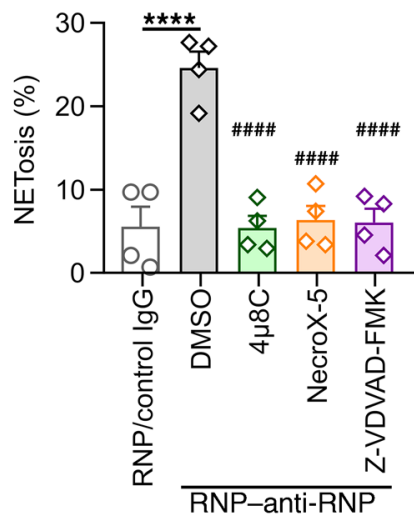

C

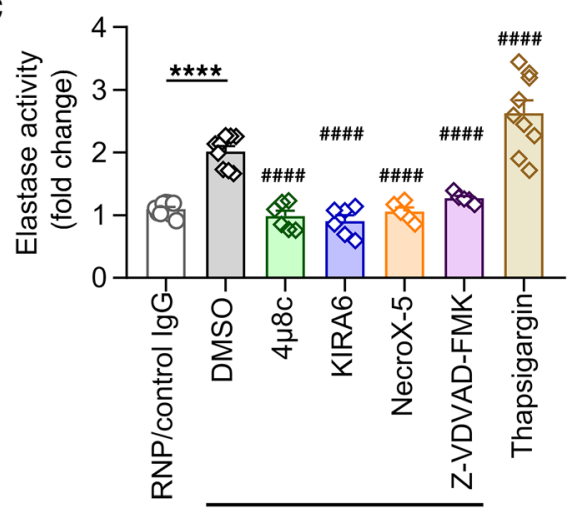

RNP-anti-RNP

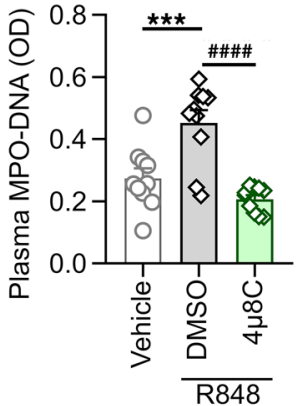

$\mathbf{F}$

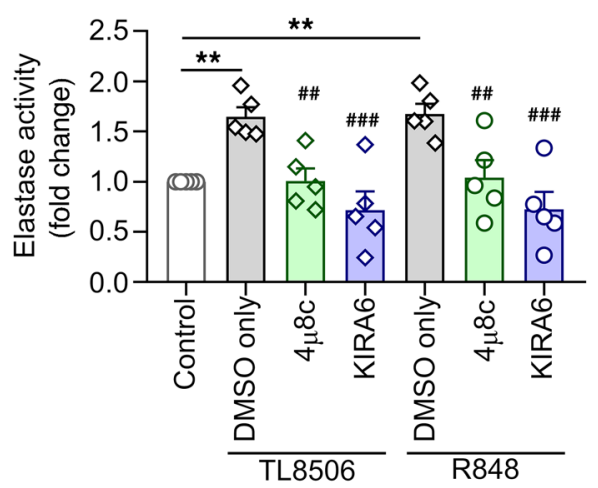

Figure 4. Inhibition of IRE1 $\alpha$ blocks lupus autoantibody-mediated NETosis. (A-D) Control neutrophils from healthy donors were stimulated with RNP-antiRNP, with or without the indicated inhibitors $(4 \mu 8 \mathrm{C}$ or KIRA6 for IRE $1 \alpha$; NecroX-5 for mitoROS; Z-VDVAD-FMK for caspase-2). The ER stress-inducing agent thapsigargin was included in some experiments as a positive control. (A) NETosis was detected after 4 hours by microscopy via staining for neutrophil elastase (red) and DNA (blue). $n=4$ independent biological replicates. ${ }^{* * *} P<0.0001$ and \#\#\#P<0.0001, compared with the RNP-anti-RNP (DMSO) group, by 1-way ANOVA followed by Holm-Sidak's multiple-comparison test. Scale bar: $50 \mu \mathrm{m}$. (B) NETosis was assayed by quantifying SYTOX Green-stained extracellular DNA. $n=5-9$ independent experiments. ${ }^{* * * *} P<0.0001$ and ${ }^{\# \# \# P} P<0.0001$, compared the with RNP-anti-RNP (DMSO) group, by 1-way ANOVA followed by Holm-Sidak's multiple-comparison test. (C) Elastase activity was assayed by quantifying the cleavage of a fluorogenic elastase substrate. $n=5-9$ independent experiments. ${ }^{* * *} P<0.0001$ and $\# \# \# P<0.0001$, compared with the RNPanti-RNP (DMSO) group, by 1-way ANOVA followed by Holm-Sidak's multiple-comparison test. (D) Plasma NETs were measured in BALB/c mice treated with R848 and the IRE1 $\alpha$ inhibitor $4 \mu 8 \mathrm{C}$, as described in Methods. $n=10$ mice per group. ${ }^{* * *} P<0.001$ and ${ }^{\# \# \#} P<0.0001$, by 1-way ANOVA followed by Holm-Sidak's multiple-comparison test, compared with the DMSO control in R848 mice. (E and $\mathbf{F}$ ) Control human neutrophils were stimulated with TL8-506 (TLR8 agonist) or R848 (TLR7/8 agonist), with or without the indicated inhibitors. (E) NETosis was assayed by quantifying SYTOX Greenstained extracellular DNA. $n=5$ independent experiments. ${ }^{* * * *} P<0.0001$,

\#\#\# $P<0.001$, and ${ }^{\# \# \# ~} P<0.0001$, compared with the RNP-anti-RNP (DMSO) control of the respective groups, by 1-way ANOVA followed by Holm-Sidak's multiple-comparison test. (F) Elastase activity was assayed by quantifying the cleavage of a fluorogenic elastase substrate. $n=$ 5 independent experiments. ${ }^{* *} P<0.01$, ${ }^{\# \#} P<0.01$, and ${ }^{\# \#} P<0.001$, compared with the RNP-anti-RNP (DMSO) control of the respective groups, by 1-way ANOVA followed by Holm-Sidak's multiple-comparison test. 
A

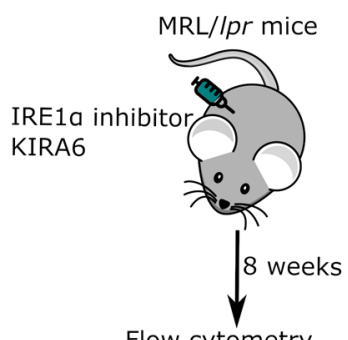

Flow cytometry antibody response

B

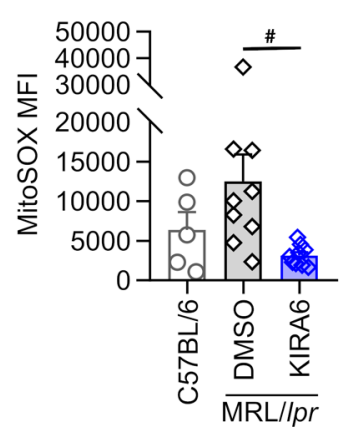

D

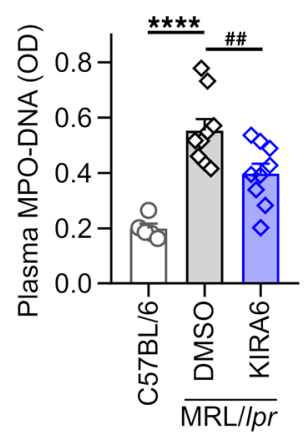

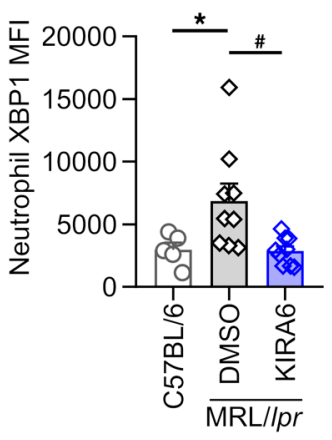

C

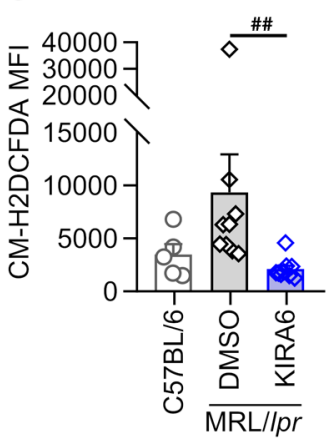

E

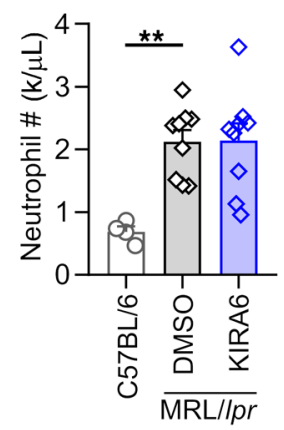

summary, these data suggest that IRE1 $\alpha$ endoribonuclease activity leads to caspase-2 activation and that there is bidirectional crosstalk between caspase-2 activation and mitoROS generation in lupus neutrophils.

IRE1a contributes to lupus-relevant NETosis. Although the above results demonstrate a role for IRE1 $\alpha$ in both ROS generation and caspase- 2 activation, we were also interested in the extent to which this pathway might contribute to neutrophil effector functions including the release of NETs (NETosis). As assessed by immunofluorescence microscopy, stimulation with RNP-anti-RNP resulted in increased NETosis compared with controls (Figure 4A), whereas pretreatment of neutrophils with IRE1 $\alpha$ inhibitors led to decreased NETosis (Figure 4A). Similarly, inhibition of either mitoROS or caspase-2 activity also reduced NETosis (Figure 4A). It should be noted that none of these inhibitors affected the viability of neutrophils outside of stimulation (Supplemental Figure 2C). Beyond microscopy, we also assayed NETosis by quantification of extracellular DNA with the cell-impermeable dye SYTOX Green. Similar to the microscopy data, we observed that NETosis was increased by RNP-anti-RNP stimula-
Figure 5. IRE1 $\alpha$ mediates neutrophil hyperactivity in lupus mice. MRL/Ipr mice were treated with the IRE1 $\alpha$ inhibitor KIRA6 as described in Methods. Each point represents an individual mouse ( $n=5$ control C57BL/ 6 and $n=9$ $\mathrm{MRL} /$ /pr mice per treatment group). After 8 weeks of treatment, peripheral blood neutrophils were analyzed by flow cytometry for (A) XBP1 protein, (B) mitoROS (MitoSOX), and (C) total cellular ROS. (D) Plasma NETs were assayed by ELISA. (E) Quantification of peripheral blood neutrophils. ${ }^{*} P<$ 0.05 , ${ }^{* *} P<0.01,{ }^{* * *} P<0.0001,{ }^{*} P<0.05$, and ${ }^{\# \# P}<0.01$, by 1 -way ANOVA followed by Holm-Sidak's multiple-comparison test (A, B, D, and E) or Kruskal-Wallis test followed by Dunn's multiple-comparison test (C).

tion and could be restrained by inhibition of IRE1 $\alpha$, mitoROS, or caspase-2 (Figure 4B). We also quantified extracellular neutrophil elastase activity as a measure of both degranulation and NETosis. In line with the other data, RNP-anti-RNP stimulation led to increased extracellular elastase activity, which decreased upon treatment with inhibitors of IRE1 $\alpha$, mitoROS, or caspase-2 (Figure $4 \mathrm{C}$ ). Interestingly, as a positive control, the known ER stress inducer thapsigargin triggered NETosis and increased elastase activity (Figure 4, B and C). In addition to IRE1 $\alpha$, we also evaluated the role of the other 2 arms of the ER stress response - the ATF6 and PERK pathways - in RNP-anti-RNP-mediated NETosis. Specifically, we quantified NETosis (SYTOX Green assay) and elastase activity in the presence of Ceapin-A7 (ATF6 inhibitor) and GSK2606414 (PERK inhibitor). Although we did not observe a decrease in NETosis upon inhibition of either PERK or ATF6 pathways (Supplemental Figure 3A), exposure to both Ceapin-A7 and GSK2606414 led to modest reductions in extracellular elastase activity (Supplemental Figure 3B).

In R848 mice, circulating plasma NETs were present at increased levels compared with controls (Figure 4D) and returned to control levels upon administration of $4 \mu 8 \mathrm{C}$ (Figure 4D). This was despite no difference in absolute neutrophil counts, lymphocyte counts, hemoglobin levels, or platelet counts (Supplemental Figure $4, \mathrm{~A}-\mathrm{D})$. Paralleling the decrease in plasma NETs, $4 \mu 8 \mathrm{C}$ also reduced the levels of total IgG and anti- $\beta$ - 2 glycoprotein I (anti- $\beta_{2} G-$ PI), but not anti-RNP or anti-dsDNA (Supplemental Figure 4, E-H).

Given these murine data, we reasoned that inhibition of IRE1 $\alpha$ might also prevent human neutrophils from releasing NETs and neutrophil elastase upon exposure to TLR7/8 agonists. Indeed, both $4 \mu 8 \mathrm{C}$ and KIRA6 (IRE1 $\alpha$ inhibitors) prevented NETosis (Figure $4 \mathrm{E}$ ) and elastase release (Figure $4 \mathrm{~F}$ ) mediated by either TL8506 (TLR8 agonist) or R848 (TLR7/8 agonist). To further confirm the role of IRE1 $\alpha$ in regulating neutrophil hyperactivity, we generated LysM ${ }^{\mathrm{Cret}} \operatorname{IRE} 1 \alpha^{\mathrm{t} / \mathrm{fl}}$ mice whose neutrophils (and other myeloid cells) lack IRE1 $\alpha$. Paralleling the experiments with human neutrophils, bone marrow neutrophils from LysM $^{\mathrm{Cre+}}$ IRE $1 \alpha^{\mathrm{A} / \mathrm{f}}$ mice and controls (LysM ${ }^{\text {Cre- }}$ IRE1 $\alpha^{\mathrm{t} / \mathrm{l}}$ mice) were stimulated with the aforementioned TLR7/8 agonists. In this context, we observed a significant reduction in NETosis (Supplemental Figure 5A) and elastase activity (Supplemental Figure 5B) when neutrophils were deficient in IRE1 $\alpha$. Furthermore, both caspase-2 activation and mitoROS formation were blunted in IRE1 $\alpha$-deficient neutrophils (Supplemental Figure 5, C and D), albeit without a full return to baseline, raising the possibility that compensatory pathways might be at play in the knockout mice. Taken together, these data implicate IRE1 $\alpha$ in lupus-relevant NETosis, both in vitro and in vivo. 
A

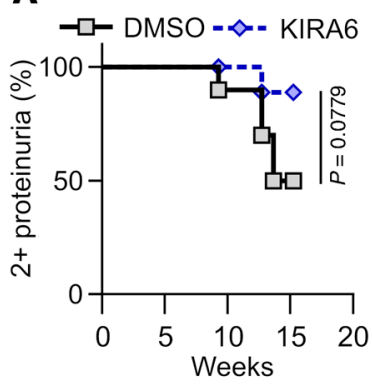

E

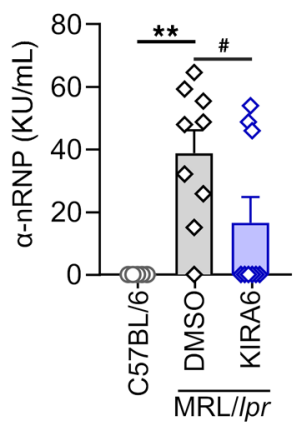

B

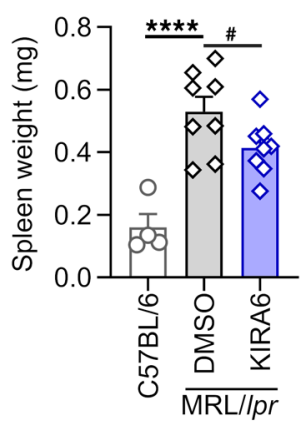

$\mathbf{F}$

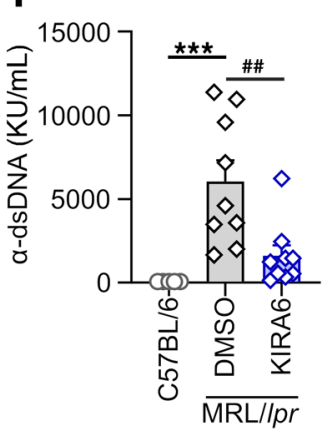

C
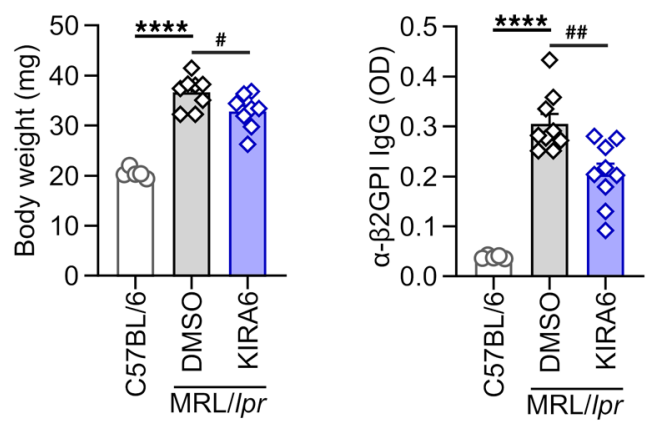

G

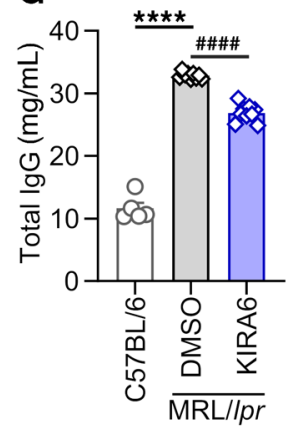

Figure 6. IRE1 $\alpha$ inhibition mitigates autoantibody responses. MRL/Ipr mice were treated with the IRE1 $\alpha$ inhibitor KIRA6 as described in Methods. $n=4-5$ control C57BL/ 6 mice and $n=8-9 \mathrm{MRL} / /$ pr mice per treatment group. (A) Proteinuria-free survival analyzed by log-rank test, (B) spleen weights, and (C) body weight after 8 weeks of treatment. (D) Plasma anti- $\beta_{2} G P I\left(\alpha-\beta_{2} G P I\right),(E)$ anti-nRNP, (F) anti-dsDNA, and (G) total IgG levels were

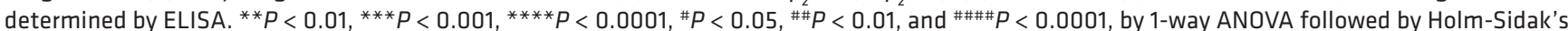
multiple-comparison test.

Inhibition of IRE1a reduces neutrophil hyperactivity in a spontaneous lupus model. MRL/lpr mice are a classic model of lupus, developing hyperactive neutrophils and lupus-associated autoantibodies by $4-5$ months of age. To delineate the role of IRE1 $\alpha$ in the development of lupus neutrophil hyperactivity, female MRL/lpr mice were treated with the IRE1 $\alpha$ inhibitor KIRA6 (5 $\mathrm{mg} / \mathrm{kg}$ /day) for a period of 8 weeks (Figure $5 \mathrm{~A}$ ). At the conclusion of the treatment period, we detected increased levels of XBP1 protein in the neutrophils of the vehicle-treated MRL/lpr mice, but not in mice that had been treated with KIRA6 (Figure $5 \mathrm{~A}$ ). Further analysis of the neutrophils revealed decreased levels of mitoROS (Figure 5B) and total ROS (Figure 5C) in the KIRA6-treated mice. We found that plasma NET levels (Figure 5D), but not absolute neutrophil numbers (Figure 5E), were also decreased by KIRA6 treatment. Taken together, these data further emphasize that inhibition of IRE1 $\alpha$ constrains neutrophil hyperactivity in the context of lupus.

Inhibition of IRE1 $\alpha$ reduces circulating autoantibody levels in $M R L / l p r$ mice. Proteinuria was measured weekly and did not differ statistically between vehicle- and KIRA6-treated MRL/lpr mice $(P=0.078$, Figure 6A). However, we detected a significant decrease in the type I IFN response in the kidneys of the KIRA6-treated mice (Supplemental Figure 6). Furthermore, KIRA6-treated mice showed significant reductions in spleen size and body weight (Figure 6, B and C). KIRA6-treated mice also had significantly lower levels of various lupus-relevant antibodies including anti- $\beta_{2} G P I$, anti-RNP, anti-dsDNA, and total IgG (Figure 6, D-G).
We also performed a comprehensive flow cytometric analysis of cell populations in the spleens and lymph nodes of MRL/lpr mice. The numbers and percentages of myeloid-lineage cells were not affected by IRE1 1 inhibition (Supplemental Figure 7). In the T cell compartment (Figure 7, A-F), we observed reduced percentages of activated $\mathrm{CD} 4^{+} \mathrm{T}$ cells $\left(\mathrm{CD} 69^{+}\right)$in both the spleen and lymph nodes (Figure 7B) as well as reduced numbers of central memory $\mathrm{CD} 4^{+}$and central memory double-negative $(\mathrm{DN}) \mathrm{T}$ cells in the lymph nodes (Figure 7, D and E). On the basis of the aforementioned reduction in autoantibody titers in MRL/lpr mice, we elected to characterize antibody-producing cells and their progenitors (Figure 8, A-D). KIRA6-treated mice had reductions in germinal center (GC) B cells $\left(\mathrm{PNA}^{+} \mathrm{GL}^{+} \mathrm{MHCII}^{+} \mathrm{B} 22 \mathrm{O}^{+}\right)$in their spleens (Figure 8B). Plasmablasts $\left(\mathrm{CD} 138^{+} \mathrm{CD} 38^{\text {lo }} \mathrm{MHCII}{ }^{+} \mathrm{B} 22 \mathrm{O}^{+}\right)$were decreased in the lymph nodes of KIRA6-treated mice (Figure 8C), whereas plasma cells (CD138 ${ }^{+}$ $\mathrm{B} 22 \mathrm{O}^{\mathrm{lo}}$ ) were decreased in both spleens and lymph nodes (Figure 8D). We observed modest reductions in activated B cells $\left(\mathrm{CD} 38^{+} \mathrm{MHCII}^{+}\right.$ $\left.\mathrm{B} 22 \mathrm{O}^{+}\right)$and increases in follicular B cells $\left(\mathrm{CD} 21^{1{ }^{\circ}} \mathrm{CD} 23^{+} \mathrm{MHCII}^{+}\right.$ $\left.\mathrm{B} 22 \mathrm{O}^{+}\right)$in the lymph nodes of KIRA6-treated mice, whereas no consistent changes in marginal zone B cells or transitional B cells were appreciated (Figure 8, E-H). In summary, these data demonstrate the potential therapeutic efficacy of IRE1 $\alpha$ inhibition in a lupus model.

\section{Discussion}

Although inhibition of IRE1 $\alpha$ has been shown to confer protection in some inflammatory models (28-30), this pathway has not been characterized in lupus neutrophils, nor has it been interro- 
A

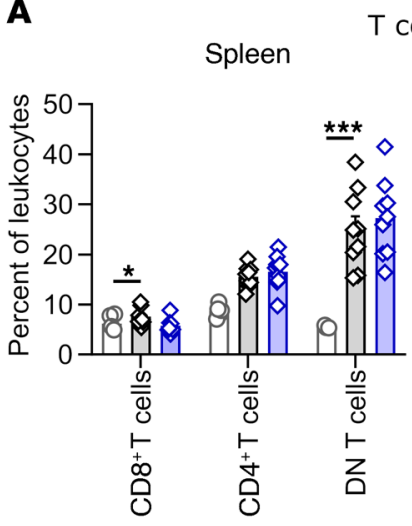

T cells

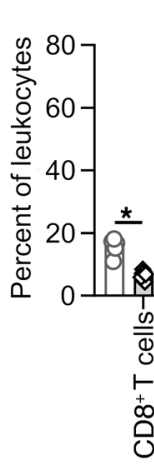

LN

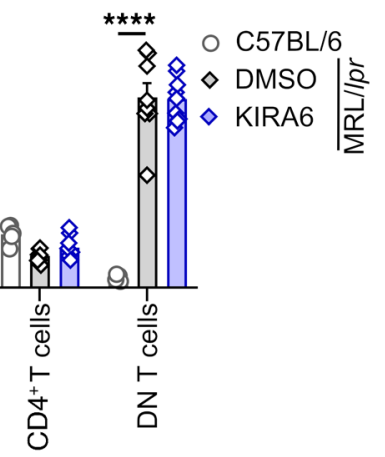

B

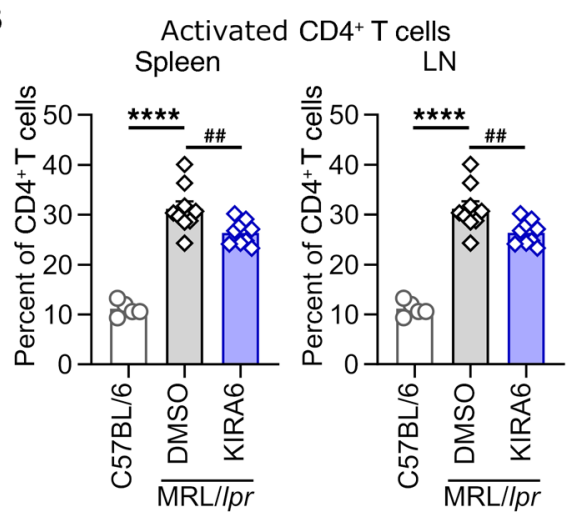

C

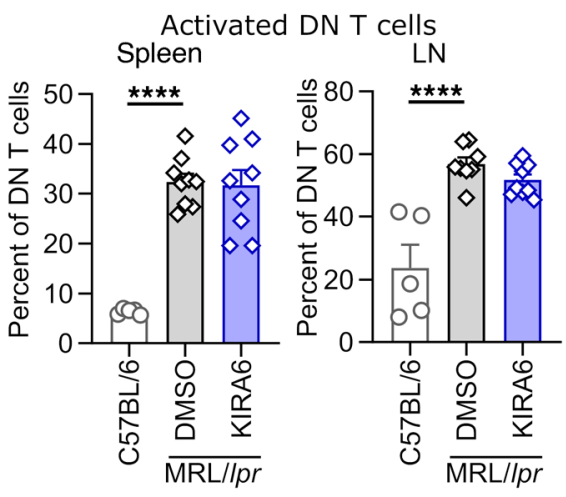

E

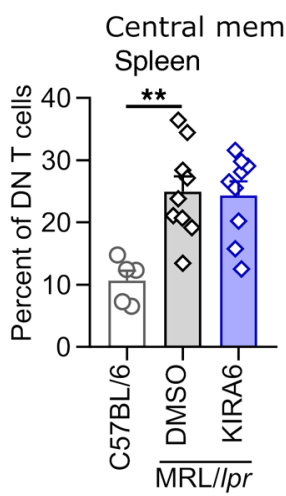

D

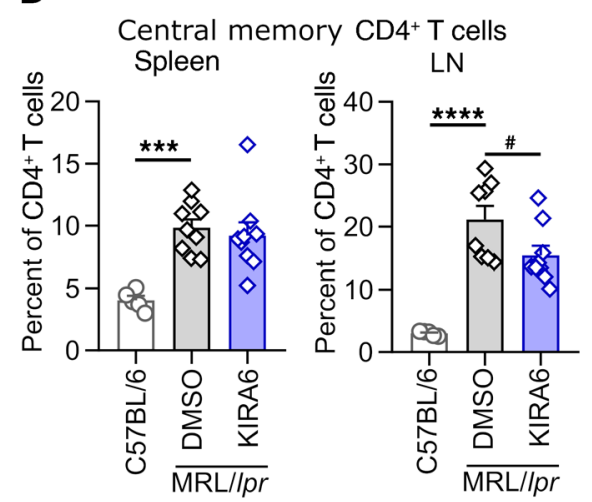

$\mathbf{F}$

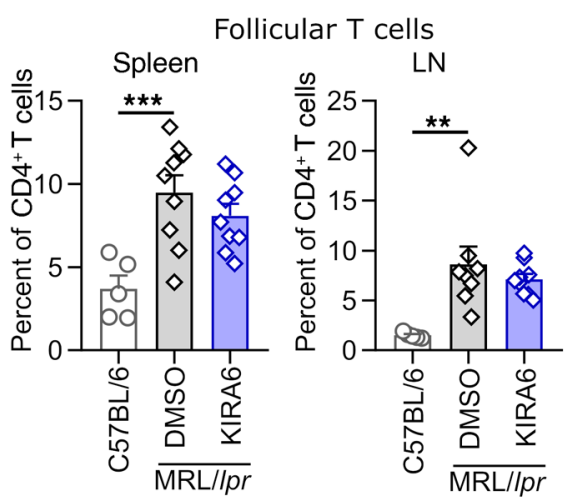

Figure 7. IRE1 $\alpha$ inhibition affects T cell activation. MRL/Ipr mice were treated with the IRE1 $\alpha$ inhibitor KIRA6 as described in Methods ( $n=4-5$ control C57BL/6 mice and $n=8-9 \mathrm{MRL} / / p r$ mice per treatment group). Splenocytes and lymph node cells were isolated after 8 weeks of treatment. (A) CD8 ${ }^{+}$, CD4+, and B220+CD4-CD8- (DN) MHCII-CD45+ T cells, (B) CD69+CD4+ T cells, (C) CD69+ DN T cells, (D) CD44+CD62L+CD4+ T cells, (E) CD44+CD62L+ DN T cells, and (F) CXCR5 ${ }^{+} P D 1^{+}{ }^{C D} 4^{+}$follicular T cells. ${ }^{*} P<0.05,{ }^{*} P<0.01,{ }^{* *} P<0.001,{ }^{* * *} P<0.0001,{ }^{*} P<0.05$, and ${ }^{\# \#} P<0.01$, by 1 -way ANOVA followed by Holm-Sidak's multiple-comparison test.

gated as a therapeutic target in lupus. Here, we have identified a heretofore unknown role for IRE1 $\alpha$ in immune complex-mediated activation of neutrophils resulting in NETosis. Analogous to what has been observed for infection-mediated macrophage activation, our results place IRE1 $\alpha$ activation upstream of mitoROS formation (35). Our studies also suggest crosstalk between caspase-2 and mitoROS in lupus neutrophils. Importantly, we found that targeting the IRE1 $\alpha / \mathrm{XBP} 1$ pathway in vivo led to reduced neutrophil mitoROS and NETosis in 2 animal models of lupus.
A variety of stimuli, including RNP-anti-RNP complexes (7), trigger NETosis via mitoROS $(16,17,36,37)$. Indeed, others have shown that RNP-anti-RNP-mediated mitoROS drive the oxidation of mitoDNA (7), which is probably a key player in downstream activation of immune responses (18). Here, we found that inhibition of the ER stress sensor IRE1 $\alpha$ neutralized mitoROS-mediated mitoDNA oxidation, supporting the idea that IRE1 $\alpha$ acts as an upstream player in mitoDNA oxidation. IRE1 $\alpha$ may be activated by TLR4, TLR7, TLR9, and other innate receptors $(22,23,38-40)$, and it is therefore 
A

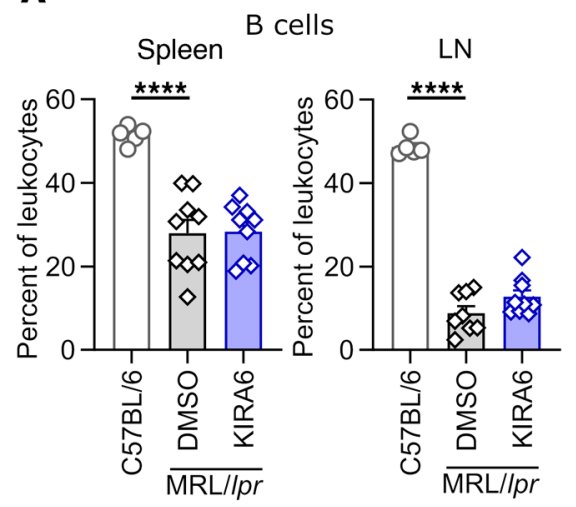

D

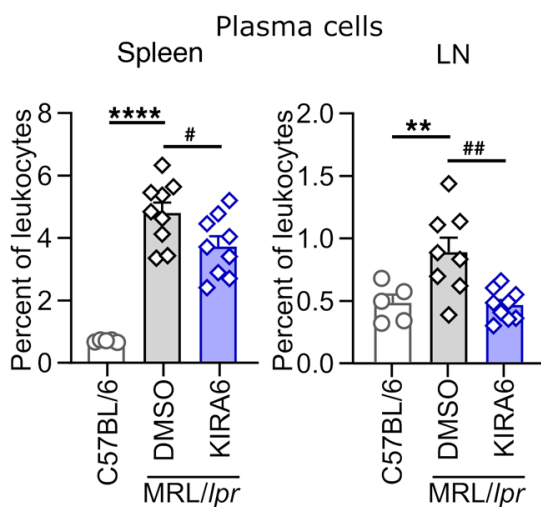

$\mathbf{F}$

$\mathrm{CD} 21^{10} \mathrm{CD} 23^{+}$follicular $\mathrm{B}$ cells

Spleen

LN
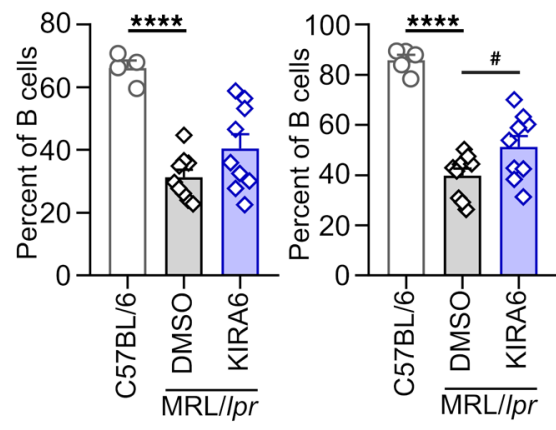

B
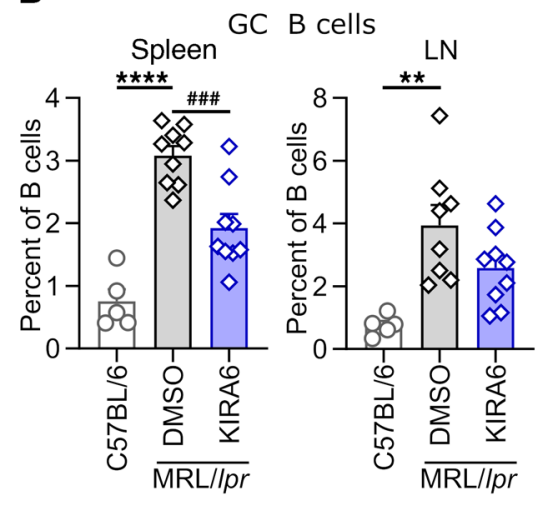

E

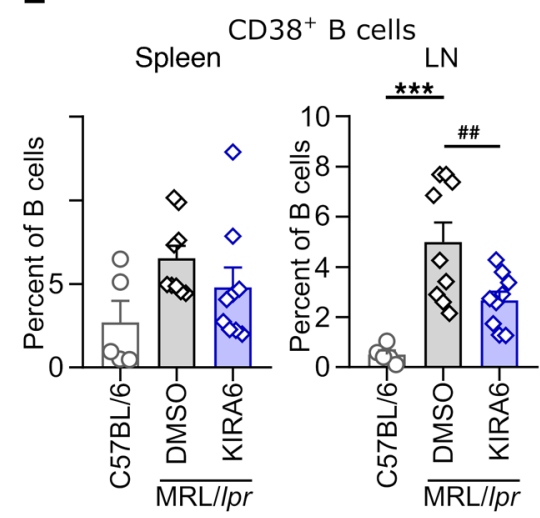

G

$\mathrm{CD} 21^{+} \mathrm{CD} 23^{\text {lo }}$ marginal zone $\mathrm{B}$ cells Spleen

$\mathrm{LN}$

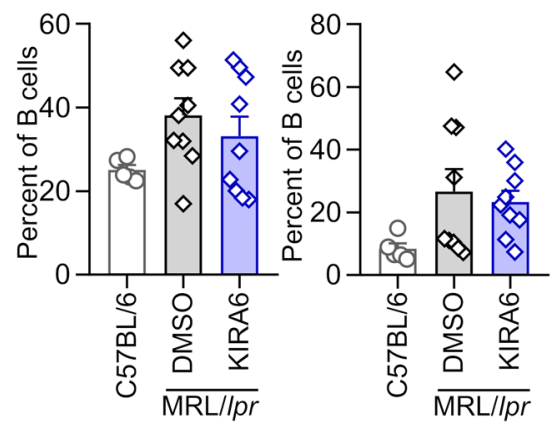

C

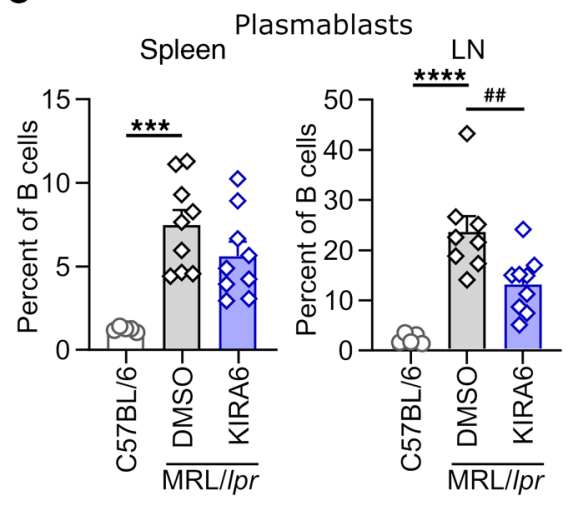

H
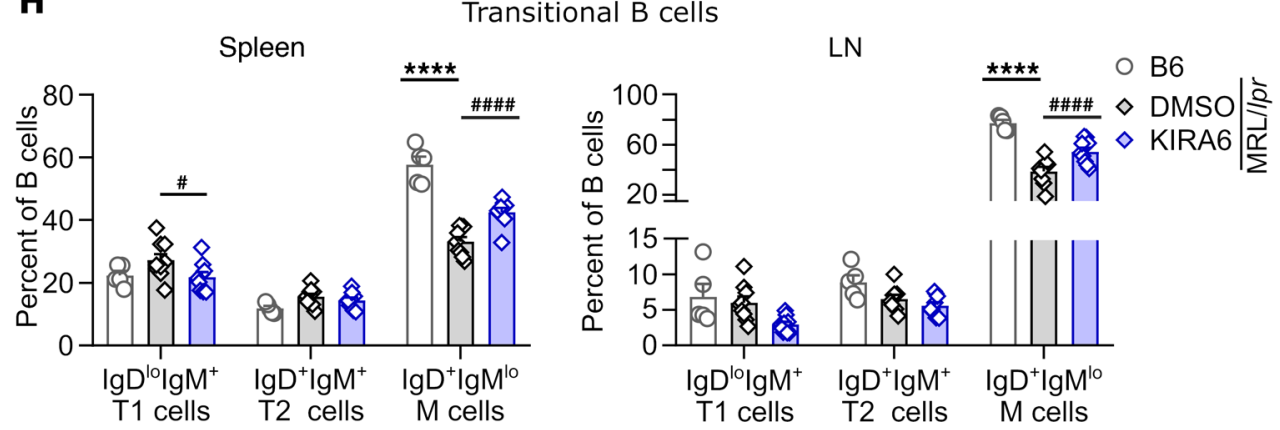

likely that RNP-anti-RNP complexes activate NETosis (41). Likewise, our data have shown that activation of TLR7 and/or TLR8 is sufficient to induce IRE1 $\alpha$-mediated NETosis. Further work will be necessary to define all aspects of the relevant signaling, and in par- ticular the mechanisms by which IRE1 $\alpha$ activation leads to mitoROS generation. One consideration is sustained calcium flux from the ER to mitochondria, which predisposes to mitoROS formation in lupus lymphocytes (42-44). Beyond calcium-mediated mechanisms, thi- 
oredoxin-interacting protein is known to shuttle in an IRE1 $\alpha$-dependent fashion to the mitochondria, where it induces mitoROS (35, 45,46 ). Along similar lines, phosphorylated JNK associates with the JNK-interacting protein Sab (SH3BP5) at the mitochondrial membrane, where it can potentiate mitoROS (47).

Our group's previous work with macrophages identified caspase- 2 as an amplifier of mitoROS, while also regulating the pore formation required for mitoDNA release (35). Initial reports pointed to caspase- 2 as a regulator of stress-mediated apoptosis (34). In that scenario, induction of ER stress may lead to activation of caspase-2 (48) and subsequently to caspase-3/7-mediated apoptosis (33). However, more recent studies have highlighted roles for caspase- 2 that are entirely independent of apoptosis. For example, a recent study of nonalcoholic steatohepatitis (NASH) in both mouse models and patients demonstrated that caspase- 2 exerts NASH-promoting effects independent of caspase-3 activation and cell death (49). There are also data to suggest that when ER stress does induce apoptosis, the process is independent of caspase-2 (50). How might caspase-2 communicate with mitoROS generation? One possibility is that ER stress-mediated induction of caspase-2 is in response to ROS-associated DNA damage. Indeed, deficiency of caspase-2 is known to increase DNA damage and genomic instability (51) and results in increased oxidative stress with aging (52). Another consideration is that increased catalytic activity of several caspases, including caspase-2, leads to protein kinase C-mediated ROS formation (53). Similarly, the IRE1 $\alpha /$ caspase-2/Bid signaling axis was recently shown to induce ROS in macrophages upon infection (35). Additional studies are needed to elucidate the apparent bidirectional relationship between mitoROS and caspase- 2 activation.

The first report linking the IRE1 $\alpha / \mathrm{XBP} 1$ pathway to lupus was published by Taguchi and colleagues, who identified XBP1 as a consistently upregulated gene in the thymic B cells of a number of mouse models of lupus including New Zealand Black, NZBxNXW $F_{1}$, and MRL/lpr (54). Todd and colleagues subsequently demonstrated that B cell-specific knockout of XBP1 protected mice from anti-dsDNA antibody production in a mouse model of lupus involving immunization with a peptide-based DNA mimotope (55). Indeed, many groups have reported that XBP1 is required for normal (and pathogenic) plasma cell differentiation (56-59), as XBP1 appears to support the growing ER mass and mitochondrial respiration that are necessary for the increased protein synthesis inherent to plasma cells (58). Along similar lines, our data demonstrate significantly reduced plasmablast and plasma cell expansion upon IRE1 $\alpha$ inhibition in lupus mouse models. In addition to plasma cells, XBP1 expression has also been detected in a small subset of cells in the light zone of GCs (59). Here, we detected a significant $(P<0.001)$ reduction in GC $B$ cells upon IRE1 $\alpha$ inhibition. As such, the reduction in lupuslike phenotypes in MRL/lpr and R848 models was likely due to the pleiotropic effects of IRE1 $\alpha$ inhibition in terms of combatting both neutrophil hyperactivity and autoreactive B cell maturation. It should also be noted that the trend toward reduced proteinuria did not reach statistical significance. As such, the extent to which the IRE1 $\alpha / \mathrm{XBP} 1$ pathway affects lupus nephritis will require future studies in these and other models with experiments that are powered to answer such questions.
Our analysis of peripheral blood neutrophils revealed increased XBP1 protein in lupus mice suggestive of IRE1 $\alpha$ activation. Importantly, we also observed increased XBP1 (spliced transcript) levels, indicative of augmented IRE1 $\alpha$ activity in neutrophils isolated from patients with lupus. These data are in line with a recent study that reported increased levels of spliced XBP1 mRNA in total leukocytes of Chinese patients with lupus (60); interestingly, the levels of IRE1 $\alpha$ itself were actually reduced in those patients. Furthermore, another recent study demonstrated increased levels of phosphorylated IRE1 $\alpha$ (without a change in the levels of total IRE1 $\alpha$ ) in mesenchymal cells of patients with lupus, again suggesting greater IRE1 $\alpha$ activity in the context of lupus (61). We should also highlight an interesting study demonstrating how ER stress may induce increased apoptosis of lupus $\mathrm{T}$ cells (62). In that scenario, the high rate of apoptosis could be symptomatic of ER stress, given that chronic or extreme ER stress causes the assembly of IRE1 $\alpha$ into high-order oligomers, resulting in the induction of a number of proapoptotic proteins that contribute to cell degeneration and death when ER stress cannot be resolved (63).

In the current study, we demonstrated IRE1 $\alpha$-mediated regulation of neutrophil hyperactivity via escalated mitoROS production and caspase-2 activation (Supplemental Figure 8). We found that pharmacological inhibition of IRE1 $\alpha$ resulted in decreased NETosis and ROS in 2 different mouse models of lupus, confirming our in vitro data on inhibitor-treated human neutrophils. Interestingly, several groups have demonstrated the beneficial effects of restraining IRE1 $\alpha$ activity in other inflammatory disease models of inflammatory arthritis, autoimmune diabetes, and atherosclerosis (28-30). The ER stress response is already being studied in the clinic as a potential treatment avenue in diabetes, with the recently approved agent liraglutide perhaps holding particular promise in this regard (64). A more precise understanding of the cellular signals that perpetuate inflammation and autoimmunity in lupus is of paramount importance as we endeavor to maximize the effectiveness and minimize the side effects of the therapies being prescribed.

\section{Methods}

Human subjects. Patients were recruited from rheumatology and hematology clinics at the University of Michigan. All patients met the American College of Rheumatology (ACR) criteria for SLE (65). SLEDAI-2K scores were prospectively calculated as described previously (66). Healthy volunteers were recruited through a posted flyer. Exclusion criteria included a history of a systemic autoimmune disease, active infection, and pregnancy. Blood was collected by phlebotomist venipuncture, and serum was prepared by standard methods and stored at $-80^{\circ} \mathrm{C}$ until use.

Preparation of human IgG and RNP-anti-RNP complexes. IgG was purified from human serum with protein $G$ agarose as described previously (67). Briefly, serum was diluted in IgG binding buffer and passed through a protein $\mathrm{G}$ agarose column (at least 5 times). Elution of $\operatorname{IgG}$ was done with $0.1 \mathrm{M}$ glycine. The solution was neutralized with $1 \mathrm{M}$ Tris, followed by overnight dialysis against PBS at $4^{\circ} \mathrm{C}$. After passing through a $0.2 \mu \mathrm{m}$ filter, IgG purity was verified by SDS-PAGE. IgG was quantified by bicinchoninic (BCA) protein assay (Pierce, Thermo Fisher Scientific). For RNP-anti-RNP complexes, IgG from 5 individuals 
with lupus and high-titer anti-RNP antibodies (all female with an average age of 39 years) was purified, pooled, mixed with SmRNP (Arotec), and used at a final concentration of $10 \mu \mathrm{g} / \mathrm{mL}$.

Human neutrophil purification. Neutrophils were isolated and purified as described previously (67). Blood from healthy volunteers was collected into heparin tubes using standard phlebotomy techniques. The anticoagulated blood was then fractionated by density-gradient centrifugation using Ficoll-Paque Plus (GE Healthcare). Neutrophils were further purified by dextran sedimentation of the red blood cell layer, before lysing residual red blood cells with water. Neutrophil preparations were at least 95\% pure as confirmed by both flow cytometry and nuclear morphology.

Mouse bone marrow neutrophil purification. Mouse bone marrow neutrophils were isolated as previously described (10). Briefly, total bone marrow cells were spun on a discontinuous Percoll gradient $(52 \%, 69 \%, 78 \%)$ at $1500 g$ for 30 minutes. Cells from the $69 \%-78 \%$ interface were then collected. These cells were $95 \% \mathrm{Ly}-6 \mathrm{G}$ positive as determined by flow cytometry and had typical nuclear morphology as shown by microscopy.

Neutrophil stimulation and inhibitor treatment. Neutrophils were conditioned with inhibitors for 30 minutes prior to stimulation with RNP-anti-RNP complexes $(10 \mu \mathrm{g} / \mathrm{mL})$ for 4 hours. The following inhibitors and reagents were used: the IRE1 $\alpha$ inhibitors $4 \mu 8 \mathrm{C}(25 \mu \mathrm{M}$, MilliporeSigma) and KIRA6 (10 $\mu \mathrm{M}$, Cayman Chemical); mitoROS scavenger NecroX-5 (10 $\mu \mathrm{M}$, Cayman Chemical); the caspase-2 inhibitor Z-VDVAD-FMK (10 $\mu \mathrm{M}$, BioVision); the ATF6 inhibitor Ceapin-A7 (10 $\mu \mathrm{M}$, MilliporeSigma); and the PERK inhibitor GSK2606414 (10 $\mu \mathrm{M}$, Sigma). Thapsigargin ( $5 \mu \mathrm{M}$, Thermo Fisher Scientific) was used as a positive control for induction of ER stress. R848 (TLR7/8 agonist, $5 \mu \mathrm{g} / \mathrm{mL}$, Enzo Life Sciences) and TL8-506 (TLR8 agonist, $500 \mathrm{ng} /$ $\mathrm{mL}$, InvivoGen) were also used for neutrophil stimulation.

$R N A$ preparation and quantitative $P C R$. At the time of tissue harvesting, kidneys were resuspended in TRIzol reagent (QIAGEN), snap-frozen in liquid nitrogen, and stored at $-80^{\circ} \mathrm{C}$. Total RNA was extracted using the Direct-zol RNA Kit (Zymo Research) according to the manufacturer's instructions, and then quantified with a BioTek Cytation5 Cell Imaging Multi-Mode Reader. cDNA synthesis was performed using the iScript cDNA Synthesis Kit (Bio-Rad). Quantitative PCR (qPCR) was performed using iTaq Universal SYBR Green Supermix (Bio-Rad) according to the manufacturer's instructions and was carried out using an ABI PRISM 7900HT (Applied Biosystems). The specific primers used are listed in Supplemental Table 1. Ct values were normalized to the housekeeping gene $U b c$.

XBP1 splicing assay. Neutrophils were pelleted and resuspended in TRIzol reagent. Total RNA was extracted, and cDNA synthesis was performed. $X B P 1$ transcripts in neutrophil cDNA were amplified using PCR Master Mix (Thermo Fisher Scientific) and the following primers: forward, 5'-AAACAGAGTAGCAGCTCAGACTGC-3' and reverse, $5^{\prime}$-CCTTCTGGGTAGACCTCTGGGAG-3'. The PCR conditions were as follows: step $1,95^{\circ} \mathrm{C}$ for 2 minutes; step $2,95^{\circ} \mathrm{C}$ for 30 seconds, $63^{\circ} \mathrm{C}$ for 30 seconds, and $72^{\circ} \mathrm{C}$ for 30 seconds for 35 amplification cycles; and step $3,72^{\circ} \mathrm{C}$ for 10 minutes. The PCR product was purified and digested with PstI to discriminate between unspliced (290 bp and $183 \mathrm{bp}$ ) and spliced (473 bp) XBP1. The digested DNA fragments were resolved by electrophoresis on a $2.5 \%$ agarose gel. Band intensities were measured using ImageJ software (NIH), and the percentage of splicing was calculated using the following formula: $\mathrm{XBP} 1 \mathrm{~s} /(\mathrm{XBP} 1 \mathrm{~s}+\mathrm{XBP} 1 \mathrm{u})$.
Immunofluorescence microscopy. Neutrophils were first adhered onto polylysine-coated $1.5 \mathrm{~mm}$ coverslips in 6-well plates. After a 30-minute pretreatment with inhibitors or control DMSO, neutrophils were stimulated with RNP-anti-RNP. After 4 hours, cells were fixed with $3.7 \%$ paraformaldehyde overnight at $4^{\circ} \mathrm{C}$ and then permeabilized with PBS plus $0.1 \%$ Triton X-100 for 15 minutes. Primary staining was performed with anti-neutrophil elastase antibody (Abcam, ab21595) and/or anti-8-OHdG antibody (StressMarq Biosciences, clone 15A3) in staining buffer (PBS plus $0.1 \%$ Triton X-100, 5\% BSA, and 10\% normal goat serum). Secondary antibodies conjugated to Alexa Fluor 594 (Jackson ImmunoResearch, 711-585-152) and Alexa Fluor 488 (Jackson ImmunoResearch, 715-545-151) were used according to the manufacturer's instructions. Coverslips were mounted using ProLong Diamond Antifade Mountant (Thermo Fisher Scientific) on microscope slides. Cells were imaged using an Olympus BX60 microscope and a BioTek Cytation5 Cell Imaging Multi-Mode Reader. All fluorescence images were processed and analyzed using ImageJ.

Plate-based assays for NETosis (SYTOX Green) and extracellular neutrophil elastase. Neutrophils were seeded onto 96-well plates. SYTOX Green (Thermo Fisher Scientific) was added to a final concentration of $0.2 \mu \mathrm{M}$, and neutrophils were treated with inhibitors and RNP-anti-RNP as above. For elastase activity, fluorogenic elastase substrate (Z-Ala-Ala-Ala-Ala)2Rh110 (50 $\mu \mathrm{M}$, Cayman Chemical) was added to the plate, and neutrophils were then treated with inhibitors and RNP-anti-RNP as above. For both assays, fluorescence was quantified at excitation and emission wavelengths of $485 \mathrm{~nm}$ and $520 \mathrm{~nm}$, respectively, using a BioTek Cytation 5 Cell Imaging Multi-Mode Reader (BioTek). NET formation was normalized to the SYTOX Green fluorescence intensity measured in the control neutrophils. Similarly, elastase activity was presented as the fold change of fluorescence intensity measured in the treated neutrophils over the intensity measured in the control group cells.

Animal housing and treatments. Mice were housed in a specific pathogen-free barrier facility and fed standard chow. C57BL/6J, $\mathrm{BALB} / \mathrm{cJ}$, LysMcre, and MRL/MpJ-Faslpr/J (MRL/lpr) mice were purchased from The Jackson Laboratory. Mice deficient in neutrophil (myeloid cell) IRE1 $\alpha$ were generated by breeding mice bearing IRE1 $\alpha$-floxed alleles (IRE1 $\alpha^{\mathrm{fl} / \mathrm{fl})}$ (68) with LysM promoter-driven Cre recombinase mice ( LysM $\left.^{\mathrm{Cre}+}\right)$. The resulting LysM $^{\mathrm{Cre+}} \mathrm{IRE} 1 \alpha^{\mathrm{fl} / \mathrm{fl}}$ mice and littermate control LysM ${ }^{\text {Cre- }}$ IRE $1 \alpha^{\mathrm{fl} / \mathrm{fl}}$ mice were used in subsequent experiments. For the induced lupus model, 8- to 10-weekold ( $n=10$ male, $n=10$ female) BALB/c mice were treated via epicutaneous application of the TLR7 agonist R848 (100 $\mu \mathrm{g} / \mathrm{mouse}$, Enzo Life Sciences) dissolved in $8 \mu \mathrm{L}$ DMSO (or DMSO alone as a control) to the right ear 3 times per week for 6 weeks. For inhibition of IRE1 $\alpha, 1$ group of mice also received a daily intraperitoneal injection of $4 \mu 8 \mathrm{C}(10 \mathrm{mg} / \mathrm{kg})$. Treatment with $4 \mu 8 \mathrm{C}$ was started on the first day of the topical R848 application. Treatment lasted for the entire duration of R848 treatment (6 weeks). In the spontaneous lupus mouse model, female MRL/lpr mice were treated with a different IRE1 $\alpha$ inhibitor, KIRA6 $(5 \mathrm{mg} / \mathrm{kg})$, or vehicle control by daily intraperitoneal injection from 8 to 16 weeks of age. KIRA6 treatment was started when mice were 8 weeks old and continued until the mice were 16 weeks old (the endpoint of the experiment). A complete blood count (CBC) from a $50 \mu \mathrm{L}$ sample of peripheral blood was provided by the In Vivo Animal Core, part of the Unit for Laboratory Animal Medicine at the University of Michigan. 
Quantification of ROS production, cytotoxicity, and caspase activation. CM-H2DCFDA (10 $\mu \mathrm{M}$, Thermo Fisher Scientific) for total cellular ROS, MitoPY1 (10 $\mu \mathrm{M}$, Tocris) for mitochondrial peroxide, and MitoSOX ( $5 \mu \mathrm{M}$, Thermo Fisher Scientific) for mitochondrial superoxide were used according to the manufacturers' instructions. The FAM-FLICA Caspase-2 Assay Kit (ImmunoChemistry Technologies) and CellEvent Caspase-3/7 Green Detection Reagent $(2 \mu \mathrm{M}$, Thermo Fisher Scientific) were used for detection of activated caspases according to the manufacturers' directions. Cell death or cytotoxicity was measured using the CellTox Green Cytotoxicity Assay (Promega). Neutrophils were washed twice with media and, where indicated, pretreated for 30 minutes with inhibitors or a control prior to stimulation. Quantification was carried out either by flow cytometry or by measurement of fluorescence at excitation and emission wavelengths of $485 \mathrm{~nm}$ and $520 \mathrm{~nm}$ (510 nm and $580 \mathrm{~nm}$ for MitoSOX) by BioTek Cytation 5 Cell Imaging Multi-Mode Reader.

Flow cytometry. Prior to staining, Fc blocking of cells was carried out using TruStain FcX (BioLegend, either human or mouse, depending on the samples) according to the manufacturer's instructions. Subsequently, cells were stained with specific antibodies for 30 minutes on ice. After washing, cells were fixed using 1-step Fix/Lyse Solution (eBioscience). Intracellular staining for XBP1s was performed using the Bioscience Foxp3/Transcription Factor Staining Buffer Set (Thermo Fisher Scientific) according to the manufacturer's protocol. Samples were analyzed on an LSRFortessa Cell Analyzer (BD Biosciences) and a Cytek Aurora (Cytek Biosciences). Further data were analyzed with FlowJo (Tree Star) and Beckman Coulter Cytobank Software (http://www.cytobank.org/). The specific antibodies used are listed in Supplemental Table 2.

ELISAs. Kits for mouse anti-dsDNA (5120), mouse anti-nRNP IgG (5415), and mouse total IgG (6320) were purchased from Alpha Diagnostic International and used according to the manufacturer's instructions. Plasma myeloperoxidase-DNA (MPO-DNA) complexes were quantified similarly to previously described methods (13). This protocol used several reagents from the Cell Death Detection ELISA kit (Roche). First, a high-binding EIA/RIA 96-well plate (Costar) was coated overnight at $4^{\circ} \mathrm{C}$ with anti-human MPO antibody (Bio-Rad, 0400-0002), diluted to a concentration of $2 \mu \mathrm{g} / \mathrm{mL}$ in coating buffer (Cell Death Detection ELISA kit). The plate was washed 3 times with wash buffer (0.05\% Tween-20 in PBS) and then blocked with 1\% BSA in PBS for 90 minutes at room temperature. The plate was washed again 3 times before incubation for 1 hour at room temperature with $1 \%$ serum or plasma in the aforementioned blocking buffer. The plate was washed 5 times and then incubated for 90 minutes at room temperature with $1 \mathrm{X}$ anti-DNA antibody (HRP-conjugated, Cell Death Detection ELISA kit) diluted 1:20 in blocking buffer. After 5 more washes, the plate was developed with $3,3^{\prime}, 5,5^{\prime}$-tetramethylbenzidine (TMB) substrate (Invitrogen, Thermo Fisher Scientific) followed by $2 \mathrm{~N}$ sulfuric acid stop solution. Absorbance was measured at a wavelength of $450 \mathrm{~nm}$ with a BioTek Cytation 5 Cell Imaging Multi-Mode Reader. For the anti- $\beta_{2}$ GPI ELISA, high-binding EIA/RIA plates (Costar) were coated overnight at $4^{\circ} \mathrm{C}$ with $10 \mu \mathrm{g} / \mathrm{mL}$ human $\beta_{2} \mathrm{GPI}$ (isolated and purified in our laboratory, ref. 69) diluted in coating buffer from the Cell Death Detection ELISA kit. Plates were then washed with $0.05 \%$ Tween-20 in PBS and blocked with 1\% BSA in PBS for 3 hours at room temperature. The plate was again washed 3 times before incubating for 1 hour at room temperature with $1 \%$ serum or plasma in the aforementioned blocking buffer. The plate was washed 5 times and then incubated for 90 minutes at room temperature with anti-mouse $\operatorname{IgG}$ antibody (HRP-conjugated, Jackson ImmunoResearch, 115-035-068) diluted 1:10,000 in blocking buffer. After 5 more washes, the plate was developed and read in the microplate reader.

Statistics. Data were analyzed with GraphPad Prism, version 7 (GraphPad Software). Normally distributed data were analyzed by $t$ test for 2 groups and by 1-way ANOVA followed by Holm-Sidak's multiple-comparison test for more than 2 groups. For skewed data, the Mann-Whitney $U$ or Kruskal-Wallis test followed by Dunn's test was used. Differences between the mean of at least 3 independent experiments are presented, with error bars showing the SEM. Statistical significance was defined as a $P$ value of less than 0.05.

Study approval. This study was reviewed and approved by the IRB of the University of Michigan. Written informed consent was received from all participants prior to inclusion in the study. Mouse experimental protocols were approved by the IACUC of the University of Michigan, and all relevant ethics regulations were followed.

\section{Author contributions}

GS, BHA, PAS, ATF, SKE, CD, and DP conducted experiments and analyzed data. JEG and JMK provided patient samples and contributed to the study design. GS, BHA, MXO, and JSK analyzed data and conceived the study. All authors participated in writing the manuscript and gave approval before submission.

\section{Acknowledgments}

The work was supported by a Target Identification in Lupus grant from the Lupus Research Alliance (to MXO and JSK). The work was also supported in part by NIH grants (R21AI13540, to MXO and R01HL134846, to JSK). JSK was partially supported by a Career Development Award from the Burroughs Wellcome Fund. GS was partially supported by a Postdoctoral Translational Scholars Program fellowship award (2UL1TR000433) from the National Center for Advancing Translational Sciences of the NIH. Additional research support was provided by the Taubman Medical Research Institute Innovation Program (to JEG and JMK) and the NIH (P3OAR075043, to JEG and JMK). JMK and JEG were also partially supported by NIH grants (R01AR071384 and NIH R01AI130025). We thank Ling Qi (University of Michigan) for providing the $\operatorname{IRE} 1 \alpha^{\mathrm{fl} / \mathrm{fl}}$ mice.

Address correspondence to: Jason S. Knight, Division of Rheumatology, Department of Internal Medicine, 1150 W. Medical Center Drive, Ann Arbor, Michigan 48109, USA. Phone: 734.936.3257; Email: jsknight@umich.edu.
1. Tsokos GC, et al. New insights into the immunopathogenesis of systemic lupus erythematosus. Nat Rev Rheumatol. 2016;12(12):716-730.

2. Brinkmann V, et al. Neutrophil extracellular traps kill bacteria. Science. 2004;303(5663):1532-1535.
3. Kenny EF, et al. Diverse stimuli engage different neutrophil extracellular trap pathways. Elife. 2017;6:e24437.

4. Knight JS, Kaplan MJ. Lupus neutrophils: 'NET' gain in understanding lupus pathogenesis. Curr
Opin Rheumatol. 2012;24(5):441-450.

5. Grayson PC, Kaplan MJ. At the bench: neutrophil extracellular traps (NETs) highlight novel aspects of innate immune system involvement in autoimmune diseases. JLeukoc Biol. 2016;99(2):253-264. 
6. Meng $\mathrm{H}$, et al. In vivo role of neutrophil extracellular traps in antiphospholipid antibody-mediated venous thrombosis. Arthritis Rheumatol. 2017;69(3):655-667.

7. Lood C, et al. Neutrophil extracellular traps enriched in oxidized mitochondrial DNA are interferogenic and contribute to lupus-like disease. Nat Med. 2016;22(2):146-153.

8. Garcia-Romo GS, et al. Netting neutrophils are major inducers of type I IFN production in pediatric systemic lupus erythematosus. Sci Transl Med. 2011;3(73):73ra20.

9. Lande R, et al. Neutrophils activate plasmacytoid dendritic cells by releasing self-DNA-peptide complexes in systemic lupus erythematosus. Sci Transl Med. 2011;3(73):73ra19.

10. Knight JS, et al. Peptidylarginine deiminase inhibition is immunomodulatory and vasculoprotective in murine lupus. J Clin Invest. 2013;123(7):2981-2993.

11. Carmona-Rivera C, et al. Neutrophil extracellular traps induce endothelial dysfunction in systemic lupus erythematosus through the activation of matrix metalloproteinase-2. Ann Rheum Dis. 2015;74(7):1417-1424.

12. Meng $\mathrm{H}$, et al. In vivo role of neutrophil extracellular traps in antiphospholipid antibody-mediated venous thrombosis. Arthritis Rheumatol. 2017;69(3):655-667.

13. Yalavarthi S, et al. Release of neutrophil extracellular traps by neutrophils stimulated with antiphospholipid antibodies: a newly identified mechanism of thrombosis in the antiphospholipid syndrome. Arthritis Rheumatol. 2015;67(11):2990-3003.

14. Van Avondt K, et al. Ligation of signal inhibitory receptor on leukocytes-1 suppresses the release of neutrophil extracellular traps in systemic lupus erythematosus. PLoS One. 2013;8(10):78459.

15. van der Linden M, et al. Neutrophil extracellular trap release is associated with antinuclear antibodies in systemic lupus erythematosus and anti-phospholipid syndrome. Rheumatology. 2018;57(7):1228-1234.

16. Wang Y, et al. Mitochondrial oxidative stress promotes atherosclerosis and neutrophil extracellular traps in aged mice. Arterioscler Thromb Vasc Biol. 2017;37(8):e99-e107.

17. Pilsczek FH, et al. A novel mechanism of rapid nuclear neutrophil extracellular trap formation in response to Staphylococcus aureus. JImmunol. 2010;185(12):7413-7425.

18. Caielli S, et al. Oxidized mitochondrial nucleoids released by neutrophils drive type I interferon production in human lupus. J Exp Med. 2016;213(5):697-713.

19. Wang H, et al. Neutrophil extracellular trap mitochondrial DNA and its autoantibody in systemic lupus erythematosus and a proof-ofconcept trial of metformin. Arthritis Rheumatol. 2015;67(12):3190-3200.

20. Navid F, Colbert RA. Causes and consequences of endoplasmic reticulum stress in rheumatic disease. Nat Rev Rheumatol. 2017;13(1):25-40.

21. Hetz C, et al. Targeting the unfolded protein response in disease. Nat Rev Drug Discov. 2013;12(9):703-719.

22. Martinon F, et al. TLR activation of the tran- scription factor XBP1 regulates innate immune responses in macrophages. Nat Immunol. 2010;11(5):411-418.

23. Beisel C, et al. TLR7-mediated activation of XBP1 correlates with the IFN $\alpha$ production in humans. Cytokine. 2017;94:55-58.

24. Genestier L, et al. TLR agonists selectively promote terminal plasma cell differentiation of $B$ cell subsets specialized in thymus-independent responses. JImmunol. 2007;178(12):7779-7786.

25. Binet F, et al. Evidence that endoplasmic reticulum (ER) stress and caspase-4 activation occur in human neutrophils. Biochem Biophys Res Commun. 2010;391(1):18-23.

26. Kuwabara WM, et al. NADPH oxidase-dependent production of reactive oxygen species induces endoplasmatic reticulum stress in neutrophil-like HL60 cells. PLoS One. 2015;10(2):0116410.

27. Hu R, et al. Endoplasmic reticulum stress of neutrophils is required for ischemia/reperfusion-induced acute lung injury. Jimmunol. 2015;195(10):4802-4809.

28. Tufanli O, et al. Targeting IRE1 with small molecules counteracts progression of atherosclerosis. Proc Natl Acad Sci U S A. 2017;114(8):E1395-E1404.

29. Qiu Q, et al. Toll-like receptor-mediated IRE1 alpha activation as a therapeutic target for inflammatory arthritis. Embo J. 2013;32(18):2477-2490.

30. Morita S, et al. Targeting ABL-IRE1alpha signaling spares ER-stressed pancreatic beta cells to reverse autoimmune diabetes. Cell Metab. 2017;25(5):1207

31. Yokogawa M, et al. Epicutaneous application of toll-like receptor 7 agonists leads to systemic autoimmunity in wild-type mice: a new model of systemic lupus erythematosus. Arthritis Rheuma tol. 2014;66(3):694-706.

32. Abuaita $\mathrm{BH}$, et al. The endoplasmic reticulum stress sensor inositol-requiring enzyme $1 \alpha$ augments bacterial killing through sustained oxidant production. mBio. 2015;6(4):e00705.

33. Cheung $\mathrm{HH}$, et al. Involvement of caspase- 2 and caspase-9 in endoplasmic reticulum stressinduced apoptosis: a role for the IAPs. Exp Cell Res. 2006;312(12):2347-2357.

34. Bouchier-Hayes L. The role of caspase- 2 in stress-induced apoptosis. J Cell Mol Med. 2010;14(6A):1212-1224.

35. Bronner DN, et al. Endoplasmic reticulum stress activates the inflammasome via NLRP3- and Caspase-2-driven mitochondrial damage. Immunity. 2015;43(3):451-462.

36. Douda DN, et al. SK3 channel and mitochondrial ROS mediate NADPH oxidase-independent NETosis induced by calcium influx. Proc Natl Acad Sci U S A. 2015;112(9):2817-2822.

37. Hosseinzadeh A, et al. Nicotine induces neutrophil extracellular traps. J Leukoc Biol. 2016;100(5):1105-1112.

38. Savic S, et al. TLR dependent XBP-1 activation induces an autocrine loop in rheumatoid arthritis synoviocytes. J Autoimmun. 2014;50(100):59-66.

39. Yao S, et al. Minimally modified low-density lipoprotein induces macrophage endoplasmic reticulum stress via toll-like receptor 4. Biochim Biophys Acta. 2012;1821(7):954-963.

40. Rivas A, et al. Targeting the unfolded protein response for disease intervention. Expert Opin
Ther Targets. 2015;19(9):1203-1218.

41. Lood C, et al. TLR7/8 activation in neutrophils impairs immune complex phagocytosis through shedding of FcgRIIA. J Exp Med. 2017;214(7):2103-2119.

42. Perl A, et al. Mitochondrial hyperpolarization: a checkpoint of T-cell life, death and autoimmunity. Trends Immunol. 2004;25(7):360-367.

43. Gergely P Jr., et al. Persistent mitochondrial hyperpolarization, increased reactive oxygen intermediate production, and cytoplasmic alkalinization characterize altered IL-10 signaling in patients with systemic lupus erythematosus. Jimmunol. 2002;169(2):1092-1101.

44. Gergely P Jr., et al. Mitochondrial hyperpolarization and ATP depletion in patients with systemic lupus erythematosus. Arthritis Rheum. 2002;46(1):175-190.

45. Saxena G, et al. Intracellular shuttling and mitochondrial function of thioredoxin-interacting protein. J Biol Chem. 2010;285(6):3997-4005.

46. Lerner AG, et al. IRE1 $\alpha$ induces thioredoxin-interacting protein to activate the NLRP3 inflammasome and promote programmed cell death under irremediable ER stress. Cell Metab. 2012;16(2):250-264

47. Chambers JW, LoGrasso PV. Mitochondrial c-Jun N-terminal kinase (JNK) signaling initiates physiological changes resulting in amplification of reactive oxygen species generation. JBiol Chem. 2011;286(18):16052-16062.

48. Upton JP, et al. IRE1 $\alpha$ cleaves select microRNAs during ER stress to derepress translation of proapoptotic Caspase-2. Science. 2012;338(6108):818-822.

49. Kim JY, et al. ER stress drives lipogenesis and steatohepatitis via caspase-2 activation of S1P. Cell. 2018;175(1):133-145.

50. Sandow JJ, et al. ER stress does not cause upregulation and activation of caspase- 2 to initiate apoptosis. Cell Death Differ. 2014;21(3):475-480.

51. Dorstyn L, et al. Caspase-2 deficiency promotes aberrant DNA-damage response and genetic instability. Cell Death Differ. 2012;19(8):1288-1298.

52. Shalini S, et al. Impaired antioxidant defence and accumulation of oxidative stress in caspase-2-deficient mice. Cell Death Differ. 2012;19(8):1370-1380.

53. Choi K, et al. Caspase-dependent generation of reactive oxygen species in human astrocytoma cells contributes to resistance to TRAIL-mediated apoptosis. Cell Death Differ. 2010;17(5):833-845.

54 . Taguchi N, et al. Increased expression of mXBP-1 (TREB-5) in thymic B cells in New Zealand mice. JAutoimmun. 2001;16(4):401-410.

55 . Todd DJ, et al. XBP1 governs late events in plasma cell differentiation and is not required for antigen-specific memory B cell development. J Exp Med. 2009;206(10):2151-2159.

56. Reimold AM, et al. Plasma cell differentiation requires the transcription factor XBP-1. Nature. 2001;412(6844):300-307.

57. Iwakoshi NN, et al. Plasma cell differentiation and the unfolded protein response intersect at the transcription factor XBP-1. Nat Immunol. 2003;4(4):321-329.

58. Shaffer AL, et al. XBP1, downstream of Blimp-1, expands the secretory apparatus and other organelles, and increases protein synthesis in plasma 
cell differentiation. Immunity. 2004;21(1):81-93. 59. Balague $\mathrm{O}$, et al. Activation of the endoplasmic reticulum stress-associated transcription factor $\mathrm{x}$ box-binding protein-1 occurs in a subset of normal germinal-center $B$ cells and in aggressive B-cell lymphomas with prognostic implications. Am J Pathol. 2009;174(6):2337-2346.

60. Wang JJ, et al. Deficiency of IRE1 and PERK signal pathways in systemic lupus erythematosus. Am J Med Sci. 2014;348(6):465-473.

61. Guo G, et al. Induction of apoptosis coupled to endoplasmic reticulum stress through regulation of CHOP and JNK in bone marrow mesenchymal stem cells from patients with systemic lupus erythematosus. J Immunol Res. 2015;2015:183738.
62. Lee WS, et al. A pathogenic role for ER stress-induced autophagy and ER chaperone GRP78/BiP in T lymphocyte systemic lupus erythematosus. JLeukoc Biol. 2015;97(2):425-433.

63. Hetz C, Papa FR. The unfolded protein response and cell fate control. Mol Cell. 2018;69(2):169-181.

64. Breton-Romero R, et al. Liraglutide treatment reduces endothelial endoplasmic reticulum stress and insulin resistance in patients with diabetes mellitus. J Am Heart Assoc. 2018;7(18):e009379.

65. Hochberg MC. Updating the American College of Rheumatology revised criteria for the classification of systemic lupus erythematosus. Arthritis Rheum. 1997;40(9):1725.
66. Gladman DD, et al. Systemic lupus erythematosus disease activity index 2000. J Rheumatol. 2002;29(2):288-291.

67. Sule G, et al. Increased adhesive potential of antiphospholipid syndrome neutrophils mediated by $\beta 2$ integrin Mac-1. Arthritis Rheumatol. 2020;72(1):114-124.

68. Sha H, et al. The ER-associated degradation adaptor protein Sel1L regulates LPL secretion and lipid metabolism. Cell Metab. 2014;20(3):458-470.

69. Ali RA, et al. Adenosine receptor agonism protects against NETosis and thrombosis in antiphospholipid syndrome. Nat Commun. 2019;10(1):1916. 